\title{
Interactions between cytoplasmic and nuclear genomes confer sex-specific effects on lifespan in Drosophila melanogaster
}

Rebecca C. Vaught*1§, Susanne Voigt*2, Ralph Dobler ${ }^{2}$, David J. Clancy ${ }^{3}$, Klaus Reinhardt**2§, Damian K. Dowling**1§

* should be considered joint first authors, ** should be considered joint senior authors, $\S_{\text {should be considered joint corresponding authors }}$

${ }^{1}$ School of Biological Sciences, Monash University, Clayton, VIC 3800, Australia ${ }^{2}$ Faculty of Biology, Applied Zoology, TU Dresden, Dresden, German

${ }^{3}$ Division of Biomedical and Life Sciences, School of Health and Medicine, Lancaster University, Lancaster LA1 4YQ, United Kingdom

\section{To whom correspondence should be addressed:}

\begin{tabular}{ll} 
Rebecca Vaught & $\begin{array}{l}\text { Damian Dowling } \\
\text { rebecca.vaught@monash.edu }\end{array}$ \\
\hline$+1(256) 924-6909$ & $\frac{\text { damian.dowling@monash.edu }}{+61(03) 9905-3864}$
\end{tabular}

Author contributions: $\mathrm{DKD}, \mathrm{RD}, \mathrm{KR}$ and RCV designed the study. RCV collected and analyzed the lifespan data. SV performed the Wolbachia screen and the genotyping analysis. RCV and DKD wrote the manuscript. All authors edited the manuscript.

Acknowledgements: We are grateful to members of the Dowling and Sgró labs for their support in the lab work associated with this project, and four anonymous reviewers for comments on a previous version of the manuscript.

Funding: Support for this experiment was provided by the Australian Research Council to DKD (DP170100165, FT160100022), the School of Biological Sciences at Monash University, and the Deutsche Forschungsgemeinschaft (Excellence Initiative to TU Dresden) to KR.

Data archival location: Sequence data is available on Genbank, SRA accession PRJNA532313: https://www.ncbi.nlm.nih.gov/sra/PRJNA532313. Phenotypic data is currently deposited on Figshare.

Running title: Cyto-nuclear interactions and the two sexes 


\section{ABSTRACT}

2 A large body of studies has demonstrated that genetic variation that resides outside of the cell

3 nucleus can affect the organismal phenotype. The cytoplasm is home to the mitochondrial

4 genome and, at least in arthropods, often hosts intracellular endosymbiotic bacteria such as

5 Wolbachia. While numerous studies have implicated epistatic interactions between

6 cytoplasmic and nuclear genetic variation as key to mediating patterns of phenotypic

7 expression, two outstanding questions remain. Firstly, the relative contribution of

8 mitochondrial genetic variation to other cytoplasmic sources of variation in shaping the

9 phenotypic outcomes of cyto-nuclear interactions remains unknown. Secondly, it remains

10 unclear whether the outcomes of cyto-nuclear interactions will manifest differently across the

11 two sexes, as might be predicted given that cytoplasmic genomes are screened by natural selection only through females as a consequence of their maternal inheritance. Here, we address these questions, creating a fully-crossed set of replicated cyto-nuclear populations derived from three geographically distinct populations of Drosophila melanogaster, and measuring the lifespan of males and females from each population. We report cyto-nuclear interactions for lifespan, with the outcomes of these interactions differing across the sexes, and reconcile these

17 findings with information on the full mitochondrial sequences and Wolbachia infection status of each of the populations.

25 Key words: mito-nuclear, mitonuclear, mtDNA, mitochondria, Wolbachia, Mother's Curse 


\section{INTRODUCTION}

27

Traditionally, most research investigating the genetic basis of variation in physiological and life-history trait expression in metazoans has focused on the role of genetic variation within the nuclear genome (Pesole et al., 2012). However, over the past two decades, it has become increasingly apparent that genetic elements that lie outside of the nuclear genome might commonly contribute to the expression of these traits. Such extra-nuclear genetic elements include the large community of microorganisms that colonise the host (such as in the digestive system), which collectively comprise the microbiome (Salvucci, 2016), several intracellular endosymbiotic bacteria, such as Buchnera (Moya et al., 2008), Orientia (Seong et al., 2001), and Wolbachia (Werren et al., 2008) that are commonly found in arthropods, as well as the genomes found within the mitochondria of virtually all contemporary eukaryotes, and the chloroplasts of plants. These extra-nuclear sources of genetic variation could in theory account for some of the documented "missing heritability" (Manolio et al., 2009) underpinning diseases and complex phenotypes, through either additive effects on the phenotype, or epistatic effects involving extra-nuclear and nuclear genetic variation.

In particular, research into the evolutionary consequences of extra-nuclear sources of genetic variation in arthropods has uncovered an unambiguous role for Wolbachia (Werren, 1997, 2008), and more recently mitochondrial genetic variation (Blier et al., 2001; Ballard \& Whitlock, 2004; Ballard \& Rand, 2005; Dowling et al., 2008; Burton et al., 2013; Dowling, 2014; Ballard \& Pichaud, 2014; Hill et al., 2019), in contributing to trait expression. The Wolbachia endosymbiotic bacterium has been shown to exert influences on the biology of its hosts, ranging from pronounced effects on the phenotype such as cytoplasmic incompatibility (CI) in some arthropod species, to neutral or minor manipulations of cellular and reproductive processes; effects hypothesized to have evolved because they increase the relative fitness of Wolbachia-infected individuals within a population (Zug \& Hammerstein, 2015; Correa \& 
that the magnitude of effects of Wolbachia infection on host phenotype can change across the nuclear genetic background of their hosts (Fry \& Rand, 2002; Fry et al., 2004; Capobianco et al., 2018), implying that epistatic interactions between Wolbachia and their hosts may contribute to the host phenotypic expression (Correa \& Ballard, 2016). was traditionally assumed to be evolutionarily neutral (Avise, 1986), many studies over the past quarter century have challenged this assumption (Ballard \& Kreitman, 1994, 1995; Rand, 2001; Dowling et al., 2008; Hill et al., 2019). Mitochondrial genetic variation has been shown to affect the expression of numerous traits spanning physiology and life-history (reviewed in Ballard \& Melvin, 2010; Horan et al., 2013; Wolff \& Gemmell, 2013; Ballard \& Pichaud, 2014; Vaught \& Dowling, 2018). The exact mechanisms underlying how this variation has been able to accumulate have not been fully characterized. It is possible that this genetic variation is comprised predominantly of nonadaptive mutations, as a result of an inefficacy of selection in shaping the genome, and debate persists as to the relative level of purifying selection which acts on the mitochondrial genome (Popadin et al., 2012; Cooper et al., 2015; James et al., 2016; Shtolz \& Mishmar, 2019). Alternatively, at least some of this genetic variation could comprise adaptive variants, and indeed several studies have recently provided evidence for this by demonstrating fitness benefits associated with particular mitochondrial variants within the environments in which they naturally exist (Blier et al., 2001; Rand, 2001; Mishmar et al., 2003; Ballard \& Whitlock, 2004; Ruiz-Pesini et al., 2004; Meiklejohn et al., 2007; Ji et al., 2012; Dowling, 2014; Camus et al., 2017). Furthermore, given that the enzyme complexes that drive mitochondrial respiration are comprised of multiple polypeptide subunits 
encoded by both the mitochondrial genome and the nuclear genome (Taanman, 1999;

77 McKenzie et al., 2007), and because many key elements of mitochondrial function are encoded by the nucleus (the genes regulating mtDNA replication, transcription, and translation machinery), a role for epistatic interactions between mitochondrial and nuclear genomes is likely to be important for maintaining cellular and ultimately organismal function (reviewed in Rand et al., 2004; Bar-Yaacov et al., 2012; Levin et al., 2014; Wolff et al., 2014; Stojković \& Đordević, 2017; Hill et al., 2019; Shtolz \& Mishmar, 2019).

(mito-nuclear) interactions in shaping the evolutionary trajectories of populations. Firstly, several studies have reported evidence that nuclear-encoded proteins targeted to the mitochondria ( $\mathrm{N}$-mt proteins) have elevated rates of evolution relative to other nuclear-encoded proteins, presumably to accompany sequence changes within the mitochondrial genome, which exhibits a higher mutation rate than the nuclear genome (Barreto and Burton, 2013; Sloan et al., 2014; Havird et al., 2015; Sloan et al., 2017; Barreto et al., 2018; Yan et al., 2019). These co-associations between evolutionary rates of mitochondrial genes and nuclear-encoded mitochondrial genes suggest that these genes are coevolving. Indeed, several studies have documented signatures of linkage disequilibrium between polymorphisms across both nuclear and mitochondrial genomes in, for instance, humans, birds, flies, nematode worms and wild carrots (Jelić et al., 2015; Sloan et al., 2015; Haddad et al., 2018; Morales et al., 2018; Adineh \& Ross, 2019; Cazares-Navarro \& Ross, 2019; Ramsey et al., 2019; but see McKenzie et al., 2019). Notwithstanding, it remains to be determined whether the mode by which these genes are coevolving is predominantly compensatory (with selection acting on nuclear polymorphisms that restore negative effects imposed by mitochondrial mutations) or synergistic (selection for optimal combinations of mitochondrial-nuclear genotype) (Sloan et al., 2017; Hill et al., 2019). Secondly, several studies have harnessed experimental designs in 
which cytoplasmic genetic components from a set of lineages have been decoupled from their putatively coevolved (or "matched") nuclear genetic backgrounds, and placed against novel (or "mismatched") backgrounds of other lineages, to create panels of strains that possess either intra-lineage "matched", or inter-lineage "mismatched" combinations of cytoplasmic and nuclear genotype. Such studies have been done by either mixing-and-matching cytoplasmic and nuclear genomes at an intra-specific (within or between populations of the same species) or interspecific (between species) scale. Such panels have been used to show that the genetic interactions between cytoplasm and nuclear genotype affect the expression of physiological and life-history traits (Wolff et al., 2014; Hill et al., 2019). This result has been substantiated by a recent meta-analysis across metazoan and plant kingdoms that reported that epistatic cytonuclear effects on trait expression tended to be larger than the additive cytoplasmic genetic effects (Dobler et al., 2014).

Furthermore, in studies that utilize cyto-nuclear strains, many of these have reported that the evolutionarily 'mismatched' combinations of mito-nuclear genotype (from different populations or even other species) exhibit lower fitness than the coevolved wild type combinations (Breeuwer \& Werren, 1995; Kenyon \& Moraes, 1997; Barrientos et al., 1998; Nagao et al., 1998; McKenzie et al., 2003; Roubertoux et al., 2003; Sackton et al., 2003; Zeyl et al., 2005; Ellison et al., 2008; Ellison \& Burton, 2008; Lee et al., 2008; Chou et al., 2010; Osada \& Akashi, 2012; Meiklejohn et al., 2013; Yee et al., 2013; Holmbeck et al., 2015; Zhu et al., 2015; Chang et al., 2016; Latorre-Pellicer et al., 2016; Ma et al., 2016; Jhuang et al., 2017; Tourmente et al., 2017; Healy \& Burton, 2019; Pichaud et al., 2019), consistent with predictions that disruption of tightly coevolved mito-nuclear complexes would lead to fitness declines (Dowling, 2008; Lane, 2011; Hill et al., 2019). However, other studies have found that while the generation of novel combinations of mitochondrial and nuclear genotype typically led to altered expression of key traits, the fitness associated with the mismatched combinations 
was not consistently lower than the fitness of matched combinations (Rand et al., 2006;

Dowling et al., 2007; Arnqvist et al., 2010; Montooth et al., 2010; Hoekstra et al., 2013; Zhu

et al., 2014; Jelić et al., 2015; Immonen et al., 2016; Mossman et al., 2016a; Hoekstra et al.,

While our knowledge of the role of cyto-nuclear interactions in the regulation of organismal phenotype in metazoans has advanced considerably over the past decade (Hill et al., 2019), a full understanding of these interactions is currently limited by certain constraints.

133 Firstly, several of the key studies conducted to date have focused on the consequences of 134 combining cytoplasmic and nuclear genotypes sourced from populations that have 135 exceptionally high levels of nucleotide divergence, or even from different species. This 136 includes studies of the copepod Tigriopus californicus, a species that exhibits up to $20 \%$ 137 divergence in mitochondrial sequences across populations (Barreto et al., 2018), and studies of 138 the nematode Caenorhabditis briggsae, which has $\sim 2.6 \%$ divergence in mitochondrial protein 139 coding genes across populations (Chang et al., 2016), as well as crosses involving 140 mitochondrial and nuclear genotypes drawn from different sub-species or species in studies of 141 mice and flies (Nagao et al., 1998; Roubertoux et al., 2003; Meiklejohn et al., 2013; Ma et al., 142 2016; Mossman et al., 2016a). While these studies provide proof-of-concept that cyto-nuclear 143 interactions can modify phenotypic expression, inferences are drawn from crosses between 144 distinct groups with high levels of mitochondrial genetic divergence, which may be 145 unrepresentative of levels typically present across populations of most animal species. 146 Moreover, studies that place cytoplasmic genomes from one species alongside highly divergent 147 nuclear backgrounds drawn from distinct species, might inadvertently unmask cryptic 148 mitochondrial genetic variation, which while adaptive or neutral when expressed alongside the 149 pool of nuclear variation with which it has coevolved, could be non-adaptive and misaligned 
to optimal fitness when expressed alongside a highly novel nuclear background to which it has interactions manifest differently across males and females, since many studies of these

154 interactions have previously focused on one sex only, or pooled the sexes in their analyses. The scope for the sex-specificity of such outcomes is particularly interesting in light of an evolutionary hypothesis called Mother's Curse, which in its broadest form proposes that the maternal inheritance of cytoplasmic genetic elements, such as the mitochondrial genome and Wolbachia genome, will lead to the accumulation of variation that is neutral or beneficial to females, but deleterious to males (Cosmides \& Tooby, 1981; Frank \& Hurst, 1996; Gemmell et al., 2004; Havird et al., 2019). Extreme examples of this include the male-killing and the conversion of males into functional females caused by certain Wolbachia among arthropods (Werren et al., 2008). Although it was long thought that similar effects would not be applicable to the streamlined genome of the bilaterian metazoan mitochondria, a number of mitochondrial variants with male-harming effects, but with no obvious negative effects or even positive effects on female function, have been identified in flies, mice, hares, and humans (Nakada et al., 2006; Xu et al., 2008; Smith et al., 2010; Clancy et al., 2011; Innocenti et al., 2011; Camus et al., 2012; Patel et al., 2016; Aw et al., 2017; Milot et al., 2017; Camus \& Dowling, 2018). The accumulation of sex-specific genetic variation in cytoplasmic genomes, particularly variation that is male-harming, may place selection on the host nuclear genome for modifier mutations that compensate for the cytoplasmically-induced effects (Turelli, 1994; Wade, 2014;

171 Ågren et al., 2018; Connallon et al., 2018). This could then lead to sex differences in the trajectories of cyto-nuclear coevolution, potentially leading to greater levels of negative epistasis for fitness in males than females upon disruption of putatively coevolved combinations of cyto-nuclear genotype, if such disruption results in the unmasking of male- 
175 harming cytoplasmic genetic variants (Dowling \& Adrian, 2019; Nagarajan-Radha et al., 2019

176

177 in-press). The results of recent studies that have partitioned cytoplasmic and nuclear genotypic contributions to the expression of adult life-history traits suggest that such a model of cytonuclear coevolution, based on nuclear modifiers rescuing the effects of male-harming cytoplasmic mutations, is plausible (Yee et al., 2013; Ma et al., 2016; Patel et al., 2016; Đorđević et al., 2015, 2017; Nagarajan-Radha et al., 2019 in-press). However, other studies investigating cyto-nuclear contributions to juvenile fitness traits and nuclear transcriptomic responses following inter-species cyto-nuclear mismatching have not found consistent malebiases (Mossman et al., 2016a, b; Mossman et al., 2017; Mossman et al., 2019).

And finally, in studies of invertebrates that utilize cyto-nuclear strains, cytoplasmic effects on the phenotype have typically been attributed to sequence variation in the mitochondrial genome, because in most cases the strains used were treated with antibiotics to eliminate concurrent infections with Wolbachia and other intracellular bacteria. However; the relative contributions of Wolbachia versus the mitochondrial genome to overall cytoplasmic effects on the phenotype currently remains unclear. Here, we further explore the capacity for cyto-nuclear interactions to confer sex-specific outcomes, by establishing a panel of fully replicated cyto-nuclear populations in D. melanogaster, in which we express cytoplasms from three geographically-distinct populations alongside representative pools of nuclear variation from each of the same three populations, in all nine possible combinations, each in triplicate. We then use these strains to investigate additive cytoplasmic, nuclear, and cyto-nuclear influences on lifespan of male and female individuals, to determine the capacity for cytonuclear interactions to manifest differently in each of the sexes, and explore the capacity for male-biases in negative epistasis upon mismatching of putatively coevolved cyto-nuclear gene complexes. We furthermore utilized a pooled-sequencing (pool-seq) approach to characterize the mitochondrial variation segregating within each of the cyto-nuclear populations, and 
determined the Wolbachia infection status of each, to enable us to explore the relative roles of

Wolbachia infection, and mitochondrial variation, in contributing to observed cyto-nuclear

202 interactions.

203

204 MATERIALS AND METHODS

\section{Construction of cyto-nuclear populations}

206 A set of fully-crossed cyto-nuclear populations of D. melanogaster was created (described also

207 in Grunau et al., (2018) and Dong et al., (2019)) using three distinct laboratory populations.

208 Each of these derived laboratory populations had originally been sourced from one of three

209 separate continents. The first from Coffs Harbour, Australia (A) was originally collected in

2102010 (Williams et al., 2012). The second population from Benin (B), Africa was originally

211 collected in 1970, is routinely used for Drosophila genetics research, and is called the Dahomey

212 population (Partridge and Andrews, 1985; Priest et al., 2007). The third population from

213 Dundas, Canada (C) was originally collected in 2005 (MacLellan et al., 2009). Each of the

214 three laboratory populations was initially founded by numerous mated-females collected from

215 their respective wild populations, and was subsequently maintained at large population sizes 216 thereafter under a 12:12 hour light: dark cycle at $25^{\circ} \mathrm{C}$ (Partridge \& Andrews, 1985; Priest et

217 al., 2007; MacLellan et al., 2009; Williams et al., 2012).

Prior to the creation of the cyto-nuclear populations used in our study, each of the three

219 populations described above was maintained as a mass-bred population, kept across 10 vials, each propagated by 15 pairs of parents, at standardized egg densities of approximately 150 eggs per vial, developing on $6 \mathrm{~mL}$ of agar-based food substrate (containing potato, yeast, and dextrose), and maintained under a $12: 12$ hour light: dark cycle at $25^{\circ} \mathrm{C}$. The offspring of each 
and Canadian populations were treated with the antibiotic tetracycline hydrochloride to remove

Wolbachia. The Coffs Harbour population remained untreated.

Our goal was to create a set of cyto-nuclear populations that captured a large and representative sample of the segregating cytoplasmic and nuclear variation from each founding laboratory population, expressing each population-representative pool of cytoplasmic variation alongside pools of segregating nuclear variation from each of the founding populations, in all nine possible combinations (three cytoplasms $[\mathrm{A}, \mathrm{B}, \mathrm{C}] \times$ three nuclear backgrounds $[\mathrm{A}, \mathrm{B}, \mathrm{C}]$ ). design explicitly sought to preserve the pool of cytoplasmic and nuclear genetic variation segregating within each of the founding populations, within each cyto-nuclear population. This, therefore, enables us to screen for interactions between population-specific pools of cytoplasmic and nuclear genetic variation. Each of the nine cyto-nuclear combinations was created independently in triplicate, which increased our capacity to statistically partition effects attributable to cytoplasmic, nuclear and cyto-nuclear effects from confounding sources of variation across the population replicates. the three laboratory populations, and separated into groups of 45. Simultaneously, 405 males of 45 females was mixed with a group of males, in all possible intra- and inter-population

244 combinations $(3 \times 3)$, with each cross combination replicated across three distinct replicates 245 (thus, 27 population crosses in total). Each group of 45 pairs of individuals was kept across three vials of 15 pairs. Ten days later, the eclosing offspring of each of the three vials per

247 combination were admixed together, and 45 virgin females collected from the admixed pool of 248 offspring. These $F_{1}$ females became the founders of a distinct cyto-nuclear population. 249 Meanwhile, 405 males were collected from each of the three original source populations, and 
again separated into groups of 45 . Each group of cyto-nuclear population females was then backcrossed to a group of males that came from the same population as the sires of these females, again across three vials of 15 pairs. In the next generations, the process was again repeated. This backcrossing procedure ran for 17 generations, resulting in nine cyto-nuclear combinations, each represented in three independent replicates (Supporting Information Fig. $99.99 \%$ of the original nuclear alleles associated with each cytoplasmic background, thus effectively placing each cytoplasm alongside the nuclear variation from the target population. The backcrossing procedure was stopped two generations prior to the experiments commencing, to enable us to expand each of the cyto-nuclear populations to numbers required to set up the experiment described below.

\section{Lifespan assay}

We assayed the adult lifespan of males and females from each cyto-nuclear population. The experiment was conducted across six sequential experimental sampling 'blocks', which were each separated in time by one generation. Males and females from each replicate cyto-nuclear population were assayed for lifespan in separate cohorts, in groups of 16 focal individuals per vial. Within each block, we assayed two cohorts of each sex for each replicate cyto-nuclear population (9 populations $\times 3$ replicates $\times 2$ sexes $\times 2$ cohorts). Each cohort of focal individuals was placed with a group of 16 "tester" individuals of the opposite sex within a given experimental vial, such that each experimental vial housed 32 individuals in total. These mixedsex conditions of even sex-ratio were employed with the aim of mimicking normal culture conditions for laboratory-adapted populations of D. melanogaster (Kimber \& Chippendale,

273 2013). Tester individuals were collected from a laboratory-adapted outbred population, $\mathrm{LH}_{\mathrm{M}}$,

274 which was originally sourced from California, U.S.A. (Rice et al., 2005). These tester 
individuals carried an autosomal recessive mutation, which had been introgressed into the

The experiment described below was implemented according to an identical protocol across each experimental block. The only difference across blocks lay in the number of generations that had elapsed since the last generation of backcrossing (three generations in

Block 1, incrementing to eight generations by Block 6). Experimental individuals and tester experimental individuals were collected under light $\mathrm{CO}_{2}$ anaesthesia from vials trimmed to approximately 150 eggs, laid by females that were five days old at the time of ovipositioning to mitigate maternal age effects (grandparental generations were also propagated by females that were five days of age at the time of ovipositioning and vials were also standardized for egg densities). Each vial used in the experiment contained a standard potato-dextrose food medium, and held 16 experimental individuals belonging to one sex and 16 tester individuals of opposite sex that were of identical age.

Once each of these experimental vials was established, each cohort was transferred to new vials with fresh food every second day, at which time the number of individuals that had died during the preceding 48 hours was scored. Expired individuals stuck to old food medium were scored for sex and eye-color, while individuals that were near-death (incapacitated but nonetheless moving) were carefully transferred to the corresponding new vial. To ensure a constant number of adult individuals (hence density) per vial, and an even sex ratio, all expired individuals (both focal individuals and tester individuals) were replaced with brown-eyed tester individuals of the same sex and similar age (within two weeks) from the $\mathrm{LH}_{\mathrm{M}-\mathrm{bw}}$ population every six days, for the first six weeks of each assay (Kimber \& Chippendale, 2013). 


\section{Mitochondrial genotyping of cyto-nuclear population}

301 The mitochondrial genomes of the cyto-nuclear populations were sequenced using a pool-seq approach, sampling 150 individuals (sexes combined) from each of the 27 population replicates. DNA was extracted by first performing a mitochondrial enrichment protocol, where

304 the individuals were first homogenized in isolation buffer (10 mM Tris-HCL, $5 \mathrm{mM}$ EDTA,

$305250 \mathrm{mM}$ sucrose, $0.2 \%$ BSA) using a $15 \mathrm{~mL}$ Dounce homogeniser on ice by eight strokes with the narrow gap pistil. The resulting supernatant was then filtrated and the homogenate was then centrifuged at $300 \mathrm{~g}$ for 3 minutes at $4^{\circ} \mathrm{C}$. To precipitate the mitochondria, the supernatant was again filtrated and a subsequent centrifugation was performed for 10 minutes at $9000 \mathrm{~g}$ at $4^{\circ}$ C. The resulting pellet was resuspended in $50 \mu \mathrm{L}$ of isolation buffer. Once mitochondria were

310 precipitated, mtDNA was extracted using the Analytic Jena innuPREP DNA Mini Kit (protocol 4), and the extracted mtDNA stored in elution buffer (Tris, $\mathrm{pH}=8$ ). The extraction yielded between $15.5 \mu \mathrm{g} / \mathrm{mL}$ and $40.1 \mu \mathrm{g} / \mathrm{mL}$ of mtDNA for each line. The mtDNA was sequenced at a concentration of $400 \mathrm{ng} / 50 \mu \mathrm{L}$ on an Illumina HiSeq platform using the 'rapid run' mode.

314 The read length was 75 bp paired ends (PE) and resulted in an average 3.05 million reads for each sample ( 150x mean sequencing depth for each line). Prior to mapping, reads were trimmed to remove low quality base calls with a minimum quality score of 20 and minimum

317 read length of 50. Reads were then mapped to the mitochondrial reference genome (NC_024511.2) using BWA v0.7.15-2 (Li, 2013). Unmapped reads were subjected to a second

319 round of mapping using Stampy v1.6.30 (Lunter \& Goodson, 2011). Subsequent quality filtering included removing reads with a mapping quality of less than 20 and duplicates. In order to detect significantly differentiated single nucleotide polymorphisms (SNPs) between the mitochondrial genomes from the cyto-nuclear populations (Australia, Benin, and Canada), the fixation index, $F_{\text {ST }}$ (Hudson et al., 1992), and Fisher's exact test for allele frequency differences were calculated per SNP in PoPoolation2 v1.201 (Kofler et al., 2011) with a 
minimum depth of 30 and a minor allele frequency of 0.01. Those SNPs that were significant

$0.1 \%$, were considered to be significantly differentiated.

\section{Wolbachia screening}

330 A screen for Wolbachia infection was performed across each of the cyto-nuclear population replicates using the approach described in Richardson et al., (2012) and Grunau et al., (2018), involving diagnostic PCR, on DNA extracted from pools of 15 adult flies, using Wolbachiaspecific wsp primer-sets (Richardson et al., 2012). This screen revealed that all populations harbouring Australian cytoplasms were infected with Wolbachia, while those possessing cytoplasms originating from Benin or Canada were uninfected.

\section{Statistical analysis}

\section{Partitioning of cytoplasmic and nuclear sources of variance}

339 The data analyses were performed in the $\mathrm{R}$ statistical environment (v3.3.3, $\mathrm{R}$ core team). We

340 first analysed whether lifespan was affected by the origin of each cytoplasm (3 levels;

341 Australia, Benin and Canada), nuclear genetic background (3 levels, Australia, Benin and

342 Canada), sex (males and females), or their interaction. We used a linear mixed effects model

343 in the lme4 package (Bates et al., 2014). The response variable was individual lifespan

344 (measured in days), with cytoplasmic origin, nuclear background, and sex included as fixed

345 factors, as well as interactions between the three. Random effects, which described the 346 hierarchical structure of the data, were also included in the model. These consisted of

347 experimental sampling block $(n=6)$, the vial identity in which the assays took place $(n=645)$,

348 and the cyto-nuclear population replicate $(n=27)$. A full model was built with all the fixed

349 effects, and including higher-order interactions involving fixed and random effects up to $2^{\text {nd }}$ 
order interactions. This full model was then reduced, by eliminating random interactions from the model that accounted for near-zero levels of variance in the model whose removal returned a non-significant $p$ value indicated by Log-Likelihood Ratio testing using the anova function in R. Parameter values of fixed effects and their significance were estimated in the final model, using Type III Wald Chi-square tests in the car package (Fox, 2012) and random effects were estimated using the summary function..

We also conducted survival analyses using mixed model Cox proportional hazard interaction. These analyses are presented in the Supporting Information (Supporting Information Table 1) and are consistent with our interpretations based on the lifespan analyses described above.

\section{Effects of cyto-nuclear mismatching}

364 We ran a second model to explicitly test whether disruption of putatively coevolved cytoplasmic and nuclear genomes led to reduced lifespan, and if so, whether any such effects were sex-biased in magnitude or direction. To this end, we included a new factor, denoted coevolutionary status of the individual ('matched' or 'mismatched' cyto-nuclear genotype), and modelled lifespan, with coevolutionary status, sex and their interactions as fixed effects. The cytoplasm of origin, nuclear background, their interaction $(3 \times 3)$, as well as the replicate cyto-nuclear population (27 levels), experimental sampling block, and vial identity were included as random effects. Once the full model was built the same model reduction process, as described above, using Log-Likelihood ratio tests in order to reduce the fixed and random effects statement, was carried out to derive at a final reduced model. This reduced model returned a model convergence warning from $\mathrm{R}$. In order to facilitate model convergence, a new 
model was constructed in which the parameter estimates for the fixed and random effects given by the original non-converging model were assigned as the starting values for the numerical algorithm for the new model with a set maximum of 20,000 iterations. Here, we present the results of the new converging model but note that the results of these two models (converging and non-converging) were virtually identical.

\section{Homing in on mitochondrial genetic effects}

The Wolbachia screen revealed that populations harbouring Australian cytoplasms were infected with Wolbachia, whereas other populations were uninfected. Furthermore, the genotyping analysis of the cyto-nuclear populations, which is reported in full in the Results, revealed that a small number of population replicates of particular cytoplasms, specifically AA3, CA1 and CA3 (notation is: Australian cytoplasm in Australian nuclear background, replicate 3; Canadian cytoplasm in Australian nuclear background, replicate 1; and Canadian cytoplasm in Australian nuclear background, replicate 3), were carrying dissimilar mitotypes (nearly fixed for different mtDNA SNPs) to the other population replicates of the same cytoplasm. In light of this, we conducted a reanalysis of the model outlined in the analysis above, excluding these outlier population replicates from the analysis. This analysis, where the outliers have been excluded, is presented in the Supporting Information (Supporting Information Table 2, Supporting Information Figure 2), and the results are qualitatively consistent with the results of the model containing all cytonuclear populations, which we present in the Results section.

\section{Partitioning mitochondrial from Wolbachia effects}

Finally, we explored the relative contributions of mitochondrial variation and heterogeneity in Wolbachia infection status in shaping the lifespan phenotype. All cyto-nuclear populations 
carrying the Australian cytoplasm were infected with Wolbachia, and thus it is challenging to

401 determine whether effects attributable to this cytoplasm are caused by Wolbachia infection, in full in the Results, one of the population replicates of the Australian cytoplasm in the

404 Australian nuclear background (AA3), is fixed for an alternative mitotype compared to the 405 other replicates of this same cyto-nuclear genotype (AA1 and AA2), and this provided the opportunity to explore whether lifespan associated with this Wolbachia-infected cytoplasm differed from that of the other Wolbachia-infected cytoplasms when expressed in the same Australian nuclear background (AA3 versus AA1 and AA2). Such a difference would point to a genuine mtDNA genotypic effect. Moreover, we compared lifespans of these three population

410 replicates (AA1, AA2, AA3) to those of the Canadian and Benin mitotypes (which are 411 Wolbachia free) when expressed in the same Australian nuclear background (CA and BA replicates). A general difference between all AA replicates and the CA and BA replicates would point to a key role for Wolbachia in driving the phenotypic effects observed in this study. Specifically, within the Australian nuclear background, there are nine population 415 replicates: three carry the Beninese cytoplasm (BA1, BA2, BA3), and as outlined in full in the 416 Results section, two carry an Australian mitotype which is dominated by a haplotype 417 previously denoted B1 (AA1, AA2), while one carries an Australian mitotype which is 418 dominated by a haplotype previously denoted A1 (AA3) (Camus et al., 2017), one carries the 419 Canadian mitotype (CA2), and CA1 and CA3 were included as their own separate grouping 420 since they carry a mitotype seemingly unique from CA2 (See Results). Thus, the fixed effects in this model included mitotype (5 levels: A1, B1, CA1\&CA3, CA2, and Benin), and sex (2 levels: males, females), and the random effects describing the hierarchical structure of the data incorporated cytoplasmic population ( 9 populations), sampling block (6 levels), and vial 424 identity (218 levels). As described above, a full model was built with all the fixed effects, 
including higher-order interactions between fixed and random effects. This full model was then reduced, by eliminating fixed and random interactions from the model that accounted for nearthe analysis of this dataset. There is no independent population replicate of the AA3 mitotype, since this was only found in one of the three Australian cytoplasm $\times$ Australian nuclear replicates (AA3). Furthermore, there is no independent population replicate of the Canadian

431 CA2 either, since the other two population replicates for this nuclear background (CA1 and aligned to the mitotype present with the populations harbouring Beninese cytoplasms.

\section{RESULTS}

\section{Mitochondrial genotyping of cyto-nuclear populations}

437 We observed 202 SNPs in the mtDNA sequences of the populations in total, of which 34 were significantly differentiated (Fisher's exact test at an FDR of $0.1 \%$ ) between the cyto-nuclear populations (Table 1). These 34 SNPs included synonymous and non-synonymous changes, as

440 well as nucleotide changes in non-coding RNAs and the origin of replication (Table 2). The majority of SNPs were significantly differentiated between the mtDNA sampled from the

442 Australian laboratory population, and those of the other two laboratory populations (Benin and

443 Canada). Genotype networks were also created based on the 34 highly differentiated SNPs and

444 consensus sequences of the 27 replicate cyto-nuclear populations (Fig. 1). The consensus 445 sequence of each population was defined by the most frequent nucleotide at each SNP site. 446 Because a pool-seq approach was utilized, it is not possible to directly determine specific

447 haplotypes. However, population-specific allele frequencies of the 34 significantly 448 differentiated SNPs were generally high (Supporting Information Fig. 3-5), making it highly 449 probable that high-frequency alleles, within each line, were found on the same haplotype. 

amount of mitochondrial genotypic variation and differentiation, relative the other cyto-nuclear

Information Fig. 3-5). The mitochondrial genetic variation within populations harbouring

454 Beninese and Canadian cytoplasms were relatively similar to each other, with only three significantly differentiated sites separating the populations (Fig. 2). The associated genotype network, based on the 34 SNPs and the consensus sequences of the 27 populations, reveals most of the populations cluster according to the location of origin of their cytoplasm (Fig. 1). more closely related to cyto-nuclear populations harbouring the Benin cytoplasm, rather than the Canadian type (Supporting Information Fig. 3-5). as distinct haplotypes (Camus et al., 2017; Lajbner et al., 2018), with one of the haplotypes, denoted A1, found to be predominate in low latitude sub-tropical regions of Australia and another haplotype denoted B1, present in higher latitude temperate regions of Australia (Camus et al., 2017). The B1 haplotype was nearly fixed (in very high frequency) in eight out of the nine cyto-nuclear populations carrying Australian cytoplasms, with the A1 haplotype nearly fixed in one population replicate (AA3, Supporting Information Fig. 3).

\section{Partitioning of cytoplasmic and nuclear sources of variance}

471 Lifespan was affected by a significant interaction between the cytoplasm, nuclear background and sex of the individuals (Table 3A, Cyto $\times$ Nuclear $\times$ Sex: $X^{2}{ }_{4}=11.150, P=0.0249$ ). The combinations of cyto-nuclear genotype that encoded longest life in females were not the same combinations that encoded longest life in males (Fig 3., Fig. 4, Supporting Information Fig. 6). 
475 For example, relative to the other two cytoplasms the Beninese cytoplasm conferred short

476 lifespan in females when expressed in the Australian nuclear background, but high relative

477 male lifespan in this same nuclear background. The Canadian cytoplasm encoded markedly

478 shorter lifespan in females when expressed in its matched Canadian nuclear background, but

479 high lifespan in males, relative to other cyto-nuclear combinations. Thus, reaction norms of

480 cytoplasm performance were discordant across sexes within the Australian and Canadian

481 nuclear backgrounds. In contrast, the reaction norms converged within the Beninese nuclear

482 background; the Canadian cytoplasm conferred the shortest lifespan in both of the sexes in this

483 background (Fig. 3, Supporting Information Fig. 6).

484 Males generally outlived females under the mixed-sex conditions in which the 485 individuals were maintained over the course of the experiment. Notably, however, the sex 486 differences in lifespan depended on the specific cyto-nuclear combination; in some cases sex 487 differences were eroded, such as the case of individuals harbouring Australian cytoplasms in the Australian nuclear background (Fig. 3A).

\section{Effects of cyto-nuclear mismatching}

491 We found no evidence that evolutionary matched (putatively 'coevolved') combinations of cyto-nuclear genotype conferred longer life than evolutionary mismatched ('mismatched') combinations, in either sex (Table 3B, Coevolutionary status: $X^{2}{ }_{1}=0.0315, P>0.05$, 494 interaction between coevolutionary status and sex was dropped from the final model: $X^{2}{ }_{l}=$ 495 0.0967, $P=0.756)$.

\section{Partitioning mitochondrial from Wolbachia effects}

498 The screen for Wolbachia revealed that all of the Australian cytoplasms are infected while the 499 others harnessed here were not. Furthermore, genotyping of the strains revealed that two of the 
population replicates of the Canadian cytoplasm with the Australian nuclear background were

501 fixed for alternative mitotypes compared to the other strains carrying Canadian cytoplasms.

502 Given this, a key consideration then becomes discerning whether the cytoplasmic effects

503 observed were primarily attributable mitochondrial sequence variation or Wolbachia infection.

504 Indeed, one of the Wolbachia-infected population replicates (AA3: Australian cytoplasm in

505 Australian nuclear background, replicate 3) carries an alternative haplotype (A1) than the other

506 Wolbachia-infected population replicates, and this provided an opportunity to explore whether

507 lifespan associated with this population differed from that of the other populations carrying

508 Australian cytoplasms within Australian nuclear background (AA1 and AA2, which harbor the

509 B1 haplotype), and also to compare the effects of the Australian cytoplasms (which are all

510 infected with Wolbachia) to those of the Canadian and Benin mitotypes (none of which are

511 infected with Wolbachia, namely BA1-3, CA1\&3, CA2) within the same Australian nuclear

512 background.

513 The interaction between mitotype and sex of the individuals was not statistically

514 significant, although the $P$ value approached the 0.05 alpha criterion (Table 4 , Mito $\times$ Sex: $X^{2}{ }_{4}$

$515=9.4313, P=0.0512$ ), and a general effect of mitotype was not found to be significant.

516 However, visual inspection of the pattern of effects across the haplotypes (Fig. 5) suggests that

517 the contrast between the mean lifespan associated with the Wolbachia-infected Australian

518 cytoplams - i.e. AA3 (which harbours the A1 haplotype) and AA1\&AA2 (which harbours the

519 B1 haplotype) - would suggest that at least some of the cytoplasmic-mediated effects described

520 above may be directly encoded by sequence differences between mitotypes.

DISCUSSION

523 The interactions between the mitochondrial and nuclear genome are key drivers of critical

524 metabolic processes in eukaryotes (Bar-Yaacov et al., 2012; Levin et al., 2014; Wolff et al., 

increasingly invoked as mediators of fundamental evolutionary processes ranging from the evolution of reproductive isolation and speciation (Gershoni et al., 2009; Chou \& Leu, 2010; Burton \& Barreto, 2012; Burton et al., 2013; Hill, 2016; Hill, 2017; Haddad et al., 2019; Lima et al., 2019; Tobler et al., 2019), to adaptation under sexual selection (Hill \& Johnson, 2013; Hill, 2015), to the evolution of sex differences (Camus et al., 2012; Vaught \& Dowling, 2018; Montooth et al., 2019), and evolution of sexual reproduction (Havird et al., 2015; reported evidence that disruption of putatively coevolved cytoplasmic and nuclear genomes leads to negative effects on phenotypic expression in eukaryotes ranging from yeast to humans (Hill et al., 2019). However, for those studies conducted in metazoans to date, many have focused on the consequences of cyto-nuclear mismatching between highly divergent genetic lineages, while others have focused on only one of the two sexes. Accordingly, it remains unclear whether the magnitude and patterns of cyto-nuclear interactions may manifest differently in each of the two sexes within the same species. the expression of lifespan across three populations of $D$. melanogaster, and to explore whether the direction or magnitude of such interactions was sex-specific. Consistent with previous

543 reports in fruit flies, copepods, other invertebrates, and mice (reviewed in Dowling et al., 2008;

544 Burton et al., 2013; Dowling, 2014; Wolff et al., 2014; Hill, 2015; Stojković \& Đordević, 2017;

545 Hill et al., 2019), we found that phenotypic expression of a key component of life-history,

546 lifespan, was contingent on epistatic interactions between cytoplasmic and nuclear genes. This

547 result is consistent with previous studies to have reported cyto-nuclear interactions for lifespan 548 and survival in fruit flies such as D. melanogaster and D. simulans (James \& Ballard, 2003; 549 Rand et al., 2006; Clancy et al., 2008; Zhu et al., 2014; Jelić et al., 2015; Drummond et al., 

interactions contribute to the expression of lifespan and that the outcomes of these interactions differ across the sexes. Our results extend on a few recent studies that have observed sexdependent mito-nuclear effects on longevity. Firstly, Drummond et al., (2019) reported that the effects of mtDNA haplotype on survival were mediated by the nuclear background in $D$. melanogaster, with evidence that the magnitude of the mito-nuclear interactions was greater in males than females. Đorđević et al., $(2015,2017)$ found these same mito-nuclear and sexspecific effects for lifespan in genetic lines of seed beetles carrying combinations of mitochondrial and nuclear genomes selected for either long or short life. Finally, Jelić et al., (2015) reported similar patterns present in Drosophila subobscura, with certain sympatric mito-nuclear combinations conferring high or low lifespan in one sex but not the other. In our study we found that, no cytoplasm conferred generally superior lifespan across nuclear backgrounds; the combinations of cyto-nuclear genotype that conferred longest life in males were not the same combinations conferring longest life in females. Furthermore, we observed no sex-specificity in the magnitude of cyto-nuclear effect size for longevity. were mediated primarily by heterogeneity in the Wolbachia infection status of the populations,

570 since Wolbachia has been observed to positively or negatively influence lifespan outcomes across previous studies (Fry \& Rand, 2002; Fry et al., 2004; Alexandrov et al., 2007;

572 Capobianco et al., 2018), or by sequence divergence in the mitochondrial genome, across the cyto-nuclear populations. To this end, we were able to address two questions. First, whether or not the major differences in lifespan across populations grouped in two clusters of cyto-nuclear 
populations - those that carry Wolbachia infection (those with cytoplasms of Australian origin)

576

577

578

579

580

581

582

583

584

585

586

587

588

589

590

591

592

593

594

595

596

597

598

599

and those that do not (all other populations). We observed that populations with the Australian

cytoplasm do not behave consistently differently relative to populations with Beninese or

Canadian cytoplasmic backgrounds, suggesting that Wolbachia is not having a disproportionately large or consistent role in their effects on lifespan. Secondly, within each cluster (Wolbachia-infected versus uninfected groups) of populations, we were able to examine whether differences in phenotypic expression across the cyto-nuclear populations mapped to the prevailing mitochondrial haplotype of each population. Specifically, we focused on the set of cyto-nuclear populations expressed in the Australian nuclear background. In this nuclear background, we found that one Wolbachia-infected cytoplasm (AA3) carried a different prevailing mitochondrial haplotype [a haplotype previously denoted A1 by Camus et al., (2017)] than the other infected cytoplasms (AA1 and AA2) that carried the B1 haplotype. The overall mean of the population carrying the B1 haplotype was putatively lower than those carrying the A1 haplotypes, and this effect seemed amplified in males (Hedges $\mathrm{g}_{\text {males }}=0.92 \pm$ 0.18 , Hedges $g_{\text {females }}=0.22 \pm 0.18$ ), albeit the interaction between haplotype and sex was not statistically significant $(P=0.053)$. The genotyping analysis indicates the $\mathrm{B} 1$ haplotype carries 20 unique SNPs that distinguish it from the Benin and Canada mitotypes (Fig. 2). Interestingly, some of these unique changes occurred within the origin of replication as well as in the tRNA Glu (Fig. 1). These genetic differences might underlie the phenotypic differences we observed, however it's difficult to confirm such a possibility in the present study.

We predicted that any cyto-nuclear incompatibilities that manifest upon experimental disruption of coevolved cyto-nuclear genotypes might exhibit signatures of male-bias. This prediction is based on the hypothesis that maternal inheritance of the mitochondrial genome will facilitate the accumulation of mutations that are male-harming, but benign or beneficial to females (Frank \& Hurst, 1996; Gemmell et al., 2004; Beekman et al., 2014; Dowling \& Adrian, 
600 2019); an evolutionary prediction that has received some empirical support from recent studies 601 in D. melanogaster (Camus et al., 2012; Innocenti et al., 2011; Patel et al., 2016; Camus \&

602 Dowling, 2018; Drummond et al., 2019; Nagarajan-Radha et al., 2019 in-press), hares (Smith 603 et al., 2010), and humans (Martikainen et al., 2017; Milot et al., 2017; Gudiseva et al., 2019).

604 We found no consistent evidence that mismatching of population-specific cytoplasmic and 605 nuclear genotypes results in a decrease in lifespan in either of the sexes. This does not exclude 606 the possibility that putatively mismatched cyto-nuclear combinations could have conferred 607 negative effects on other key traits not assessed in this study, such as reproduction, metabolic 608 rate or ROS production. However, the results are consistent with the idea that the levels of 609 mitochondrial genetic divergence observed within many species may not be sufficient to drive 610 mito-nuclear mediated reproductive isolation between populations, a plausible outcome in the 611 face of gene flow between these populations. Such a pattern would be congruent with a 612 growing body of similar studies conducted at the intra-specific scale, in Drosophila and 613 Callosobruchus beetles, which have reported that although cyto-nuclear interactions are 614 present, the experimental mismatching of putatively coevolved combinations does not 615 consistently lead to poorer phenotypic outcomes (Dowling et al., 2007; Arnqvist et al., 2010; 616 Jelić et al., 2015). Notwithstanding, some studies examining the consequences of mito-nuclear 617 mismatching at the intraspecific scale have documented clear evidence for fitness impairment 618 in some species; albeit these studies exhibit much higher levels of mtDNA sequence divergence 619 than seen between populations of humans or D. melanogaster (Morrow et al., 2015). This 620 includes studies of the intertidal copepod T. californicus, which exhibits mtDNA sequence 621 divergence of up to $20 \%$ between populations, and the nematode $C$. briggsae, which exhibits 622 divergence of around 2.6\% for protein-coding genes (Chang et al., 2016; Haddad et al., 2018; 623 Adineh \& Ross, 2019; Cazares-Navarro \& Ross, 2019). Currently, however, it remains unclear 624 whether increases in levels of mtDNA sequence divergence between populations are likely to 
routinely drive the emergence of mito-nuclear incompatibilities; since several studies comparing combinations of mito-nuclear genotype sourced from different species of combinations (Zhu et al., 2014; Mossman et al., 2016a, b; Mossman et al., 2019). As such, the

630 likelihood of mito-nuclear incompatibility occurring, or the degree of mito-nuclear incompatibility, may not necessarily correspond to the amount of genetic divergence present, et al., 2019). In conclusion, our study provides some new insights into the dynamics and evolutionary implications of cyto-nuclear interactions, by showing cyto-nuclear epistasis contributes to lifespan even at the intraspecies scale, and that the magnitude and pattern of effects across cytonuclear combination can be sex-specific. This suggests that selection for optimally performing cyto-nuclear genotypes will in part be sex-specific, which is likely to be the case given the mitochondrial genome is transmitted only through females, and can, therefore, only respond to

640 selection directly through females. Currently, several questions remain. It is unclear how much of the cyto-nuclear effect on life-history trait expression is explicitly tied to mitochondrial sequence divergence; or how much mitochondrial divergence is required to precipitate fitness

643 interactions with the nuclear genotype. Recently, it has even be argued that cyto-nuclear

644 interactions will be relevant to molecular processes that are at play within the individual 645 (Havird et al., 2019; Shtolz \& Mishmar, 2019; Wei et al., 2019), even occurring via interactions between gene products encoded by different mtDNA molecules, present in heteroplasmy

647 within the cell, and nuclear-encoded products (Wolff et al., 2014), and to epigenetic 648 mechanisms (Grunau et al., 2018; Kopinski et al., 2019). Ultimately, the degree to which such 649 interactions may contribute to the missing variance in quantitative genetic studies remains to 
650 be fully investigated (Rand et al., 2018; Zhu et al., 2019). Finally, it is now clear that several extra-nuclear sources of variance may affect the expression of the organismal phenotype, and

652 it is possible that these cytoplasmic genetic elements interact not only with genetic variants in

653 the nuclear background to shape trait values via cyto-nuclear interactions, but also epistatically

654 with each other, which could manifest in complex cytoplasmic-by-cytoplasmic-by-nuclear

655 interactions underpinning variation in organismal function (Labjner et al., 2018; Havird et al.,

656 2019). Such possibilities deserve experimental attention.

657

658

659

\section{Conflict of interest statement:}

660

661

All authors declare no conflict of interest

662

663

664

665

666

667

668

669

670

671

672

673

674

675

676

677 


\section{REFERENCES}

Adineh, S., \& Ross, J. (2019). The ebb and flow of heteroplasmy during intra-species hybridization in Caenorhabditis briggsae. bioRxiv, 623207. https://doi.org/10.1101/623207

Ågren, J. A., Munasinghe, M., \& Clark, A. G. (2019). Sexual conflict through mother's curse and father's curse. Theoretical Population Biology. https://doi.org/10.1016/j.tpb.2018.12.007

Alexandrov, I. D., Alexandrova, M. V., Goryacheva, I. I., Rochina, N. V., Shaikevich, E. V., \& Zakharov, I. A. (2007). Removing endosymbiotic Wolbachia specifically decreases lifespan of females and competitiveness in a laboratory strain of Drosophila melanogaster. Russian Journal of Genetics, 43(10), 1147-1152. https://doi.org/10.1134/S1022795407100080

Arnqvist, G., Dowling, D. K., Eady, P., Gay, L., Tregenza, T., Tuda, M., \& Hosken, D. J. (2010). Genetic architecture of metabolic rate: environment specific epistasis between mitochondrial and nuclear genes in an insect. Evolution, 64(12), 3354-3363. https://doi.org/10.1111/j.1558-5646.2010.01135.x

Avise, J. C. (1986). Mitochondrial DNA and the evolutionary genetics of higher animals. Philosophical Transactions of the Royal Society of London. B, Biological Sciences, 312(1154), 325-342. https://doi.org/10.1098/rstb.1986.0011

Aw, W. C., Garvin, M. R., Melvin, R. G., \& Ballard, J. W. O. (2017). Sex-specific influences of mtDNA mitotype and diet on mitochondrial functions and physiological traits in Drosophila melanogaster. PloS one, 12(11), e0187554. https://doi.org/10.1371/journal.pone.0187554

Ballard, J. W. O., \& Melvin, R. G. (2010). Linking the mitochondrial genotype to the organismal phenotype. Molecular Ecology, 19(8), 1523-1539. https://doi.org/10.1111/j.1365-294X.2010.04594.X

Ballard, J. W. O., \& Pichaud, N. (2014). Mitochondrial DNA: more than an evolutionary bystander. Functional Ecology, 28(1), 218-231. https://doi.org/10.1111/13652435.12177

Ballard, J. W. O., \& Rand, D. M. (2005). The population biology of mitochondrial DNA and its phylogenetic implications. Annual Review of Ecology, Evolution, and Systematics, 36, 621-642. https://doi.org/10.1146/annurev.ecolsys.36.091704.175513

Ballard, J. W., \& Kreitman, M. (1994). Unraveling selection in the mitochondrial genome of Drosophila. Genetics, 138(3), 757-772.

Ballard, J. W. O., \& Kreitman, M. (1995). Is mitochondrial DNA a strictly neutral marker?. Trends in Ecology \& Evolution, 10(12), 485-488. https://doi.org/10.1016/S01695347(00)89195-8

Ballard, J. W. O., \& Whitlock, M. C. (2004). The incomplete natural history of mitochondria. Molecular Ecology, 13(4), 729-744. https://doi.org/10.1046/j.1365294X.2003.02063.X

Bar-Yaacov, D., Blumberg, A., \& Mishmar, D. (2012). Mitochondrial-nuclear co-evolution and its effects on OXPHOS activity and regulation. Biochimica et Biophysica Acta (BBA)-Gene Regulatory Mechanisms, 1819(9-10), 1107-1111. https://doi.org/10.1016/j.bbagrm.2011.10.008

Barreto, F. S., \& Burton, R. S. (2013). Elevated oxidative damage is correlated with reduced fitness in interpopulation hybrids of a marine copepod. Proceedings of the Royal Society B: Biological Sciences, 280(1767), 20131521. https://doi.org/10.1098/rspb.2013.1521 
Barreto, F. S., Watson, E. T., Lima, T. G., Willett, C. S., Edmands, S., Li, W., \& Burton, R. S. (2018). Genomic signatures of mitonuclear coevolution across populations of Tigriopus californicus. Nature Ecology \& Evolution, 2(8), 1250. https://doi.org/10.1038/s41559-018-0588-1

Barrientos, A., Kenyon, L., \& Moraes, C. T. (1998). Human xenomitochondrial cybrids cellular models of mitochondrial complex I deficiency. Journal of Biological Chemistry, 273(23), 14210-14217. https://doi.org/10.1074/jbc.273.23.14210

Bates, D., Mächler, M., Bolker, B., \& Walker, S. (2014). Fitting linear mixed-effects models using lme4. arXiv preprint arXiv:1406.5823.

Beekman, M., Dowling, D. K., \& Aanen, D. K. (2014). The costs of being male: are there sex-specific effects of uniparental mitochondrial inheritance?. Philosophical Transactions of the Royal Society B: Biological Sciences, 369(1646), 20130440. https://doi.org/10.1098/rstb.2013.0440

Benjamini, Y., \& Hochberg, Y. (1995). Controlling the false discovery rate: a practical and powerful approach to multiple testing. Journal of the Royal Statistical Society: Series B (Methodological), 57(1), 289-300. https://doi.org/10.1111/j.25176161.1995.tb02031.x

Blier, P. U., Dufresne, F., \& Burton, R. S. (2001). Natural selection and the evolution of mtDNA-encoded peptides: evidence for intergenomic co-adaptation. TRENDS in Genetics, 17(7), 400-406. https://doi.org/10.1016/S0168-9525(01)02338-1

Breeuwer, J. A., \& Werren, J. H. (1995). Hybrid breakdown between two haplodiploid species: the role of nuclear and cytoplasmic genes. Evolution, 49(4), 705-717. https://doi.org/10.1111/j.1558-5646.1995.tb02307.x

Burton, R. S., \& Barreto, F. S. (2012). A disproportionate role for mtDNA in DobzhanskyMuller incompatibilities?. Molecular Ecology, 21(20), 4942-4957. https://doi.org/10.1111/mec.12006

Burton, R. S., Pereira, R. J., \& Barreto, F. S. (2013). Cytonuclear genomic interactions and hybrid breakdown. Annual Review of Ecology, Evolution, and Systematics, 44, 281 302. https://doi.org/10.1146/annurev-ecolsys-110512-135758

Camus, M. F., Clancy, D. J., \& Dowling, D. K. (2012). Mitochondria, maternal inheritance, and male aging. Current Biology, 22(18), 1717-1721. https://doi.org/10.1016/j.cub.2012.07.018

Camus, M. F., \& Dowling, D. K. (2018). Mitochondrial genetic effects on reproductive success: signatures of positive intrasexual, but negative intersexual pleiotropy. Proceedings of the Royal Society B: Biological Sciences, 285(1879), 20180187. https://doi.org/10.1098/rspb.2018.0187

Camus, M. F., Wolff, J. N., Sgro, C. M., \& Dowling, D. K. (2017). Experimental support that natural selection has shaped the latitudinal distribution of mitochondrial haplotypes in Australian Drosophila melanogaster. Molecular Biology and Evolution, 34(10), 2600-2612. https://doi.org/10.1093/molbev/msx 184

Capobianco III, F., Nandkumar, S., \& Parker, J. D. (2018). Wolbachia affects survival to different oxidative stressors dependent upon the genetic background in Drosophila melanogaster. Physiological Entomology, 43(3), 239-244. https://doi.org/10.1111/phen.12252

Cazares-Navarro, E., \& Ross, J. A. (2019). Temperature-dependent mitochondrial-nuclear epistasis. microPublication Biology. https://doi.org/10.17912/micropub.biology.000147

Chang, C. C., Rodriguez, J., \& Ross, J. (2016). Mitochondrial-nuclear epistasis impacts fitness and mitochondrial physiology of interpopulation Caenorhabditis briggsae 
hybrids. G3: Genes, Genomes, Genetics, 6(1), 209-219. https://doi.org/10.1534/g3.115.022970

Chevin, L. M., \& Hoffmann, A. A. (2017). Evolution of phenotypic plasticity in extreme environments. Philosophical Transactions of the Royal Society B: Biological Sciences, 372(1723), 20160138. https://doi.org/10.1098/rstb.2016.0138

Chou, J. Y., Hung, Y. S., Lin, K. H., Lee, H. Y., \& Leu, J. Y. (2010). Multiple molecular mechanisms cause reproductive isolation between three yeast species. PLoS biology, 8(7), e1000432. https://doi.org/10.1371/journal.pbio.1000432

Chou, J. Y., \& Leu, J. Y. (2010). Speciation through cytonuclear incompatibility: insights from yeast and implications for higher eukaryotes. Bioessays, 32(5), 401-411. https://doi.org/10.1002/bies.200900162

Clancy, D. J. (2008). Variation in mitochondrial genotype has substantial lifespan effects which may be modulated by nuclear background. Aging Cell, 7(6), 795-804. https://doi.org/10.1111/j.1474-9726.2008.00428.x

Clancy, D. J., Hime, G. R., \& Shirras, A. D. (2011). Cytoplasmic male sterility in Drosophila melanogaster associated with a mitochondrial CYTB variant. Heredity, 107(4), 374. https://doi.org/10.1038/hdy.2011.12

Connallon, T., Camus, M. F., Morrow, E. H., \& Dowling, D. K. (2018). Coadaptation of mitochondrial and nuclear genes, and the cost of mother's curse. Proceedings of the Royal Society B: Biological Sciences, 285(1871), 20172257. https://doi.org/10.1098/rspb.2017.2257

Cooper, B. S., Burrus, C. R., Ji, C., Hahn, M. W., \& Montooth, K. L. (2015). Similar efficacies of selection shape mitochondrial and nuclear genes in both Drosophila melanogaster and Homo sapiens. G3: Genes, Genomes, Genetics, 5(10), 2165-2176. https://doi.org/10.1534/g3.114.016493

Correa, C. C., \& Ballard, J. W. O. (2016). Wolbachia associations with insects: winning or losing against a master manipulator. Frontiers in Ecology and Evolution, 3, 153. https://doi.org/10.3389/fevo.2015.00153

Cosmides, L. M., \& Tooby, J. (1981). Cytoplasmic inheritance and intragenomic conflict. Journal of Theoretical Biology, 89(1), 83-129. https://doi.org/10.1016/00225193(81)90181-8

Dobler, R., Dowling, D. K., Morrow, E. H., \& Reinhardt, K. (2018). A systematic review and meta-analysis reveals pervasive effects of germline mitochondrial replacement on components of health. Human Reproduction Update, 24(5), 519-534. https://doi.org/10.1093/humupd/dmy018

Dobler, R., Rogell, B., Budar, F., \& Dowling, D. K. (2014). A meta-analysis of the strength and nature of cytoplasmic genetic effects. Journal of evolutionary biology, 27(10), 2021-2034. https://doi.org/10.1111/jeb.12468

Đorđević, M., Savković, U., Lazarević, J., Tucić, N., \& Stojković, B. (2015). Intergenomic interactions in hybrids between short-lived and long-lived lines of a seed beetle: Analyses of life history traits. Evolutionary Biology, 42(4), 461-472. https://doi.org/10.1007/s11692-015-9340-9

Đorđević, M., Stojković, B., Savković, U., Immonen, E., Tucić, N., Lazarević, J., \& Arnqvist, G. (2017). Sex-specific mitonuclear epistasis and the evolution of mitochondrial bioenergetics, ageing, and life history in seed beetles. Evolution, 71(2), 274-288. https://doi.org/10.1111/evo.13109

Dong, W., Dobler, R., Dowling, D. K., \& Moussian, B. (2019). The cuticle inward barrier in Drosophila melanogaster is shaped by mitochondrial and nuclear genotypes and a sex-specific effect of diet. PeerJ, 7, e7802. https://doi.org/10.7717/peerj.7802 
Dowling, D. K., Meerupati, T., \& Arnqvist, G. (2010). Cytonuclear interactions and the economics of mating in seed beetles. The American Naturalist, 176(2), 131-140. https://doi.org/10.1086/653671

Dowling, D. K. (2014). Evolutionary perspectives on the links between mitochondrial genotype and disease phenotype. Biochimica Et Biophysica Acta (BBA)-General Subjects, 1840(4), 1393-1403. https://doi.org/10.1016/j.bbagen.2013.11.013

Dowling, D. K., Abiega, K. C., \& Arnqvist, G. (2007). Temperature-specific outcomes of cytoplasmic-nuclear interactions on egg-to-adult development time in seed beetles. Evolution, 61(1), 194-201. https://doi.org/10.1111/j.1558-5646.2007.00016.x

Dowling, D. K., \& Adrian, R. E. (2019). Challenges and prospects for testing the Mother's Curse hypothesis. Integrative and Comparative Biology. https://doi.org/10.1093/icb/icz110

Dowling, D. K., Friberg, U., \& Lindell, J. (2008). Evolutionary implications of non-neutral mitochondrial genetic variation. Trends in Ecology \& Evolution, 23(10), 546-554. https://doi.org/10.1016/j.tree.2008.05.011

Ellison, C. K., \& Burton, R. S. (2008). Interpopulation hybrid breakdown maps to the mitochondrial genome. Evolution, 62(3), 631-638. https://doi.org/10.1111/j.15585646.2007.00305.x

Ellison, C. K., Niehuis, O., \& Gadau, J. (2008). Hybrid breakdown and mitochondrial dysfunction in hybrids of Nasonia parasitoid wasps. Journal of Evolutionary Biology, 21(6), 1844-1851. https://doi.org/10.1111/j.1420-9101.2008.01608.x

Fox, J., Weisberg, S., Adler, D., Bates, D., Baud-Bovy, G., Ellison, S., ... \& Heiberger, R. (2012). Package 'car'. Vienna: R Foundation for Statistical Computing.

Frank, S. A., \& Hurst, L. D. (1996). Mitochondria and male disease. Nature, 383(6597), 224. https://doi.org/10.1038/383224a0

Friberg, U., \& Dowling, D. K. (2008). No evidence of mitochondrial genetic variation for sperm competition within a population of Drosophila melanogaster. Journal of Evolutionary Biology, 21(6), 1798-1807. https://doi.org/10.1111/j.14209101.2008.01581.x

Fry, A. J., Palmer, M. R., \& Rand, D. M. (2004). Variable fitness effects of Wolbachia infection in Drosophila melanogaster. Heredity, 93(4), 379. https://doi.org/10.1038/sj.hdy.6800514

Fry, A. J., \& Rand, D. M. (2002). Wolbachia interactions that determine Drosophila melanogaster survival. Evolution, 56(10), 1976-1981. https://doi.org/10.1111/j.00143820.2002.tb00123.x

Gemmell, N. J., Metcalf, V. J., \& Allendorf, F. W. (2004). Mother's curse: the effect of mtDNA on individual fitness and population viability. Trends in Ecology \& Evolution, 19(5), 238-244. https://doi.org/10.1016/j.tree.2004.02.002

Gershoni, M., Templeton, A. R., \& Mishmar, D. (2009). Mitochondrial bioenergetics as a major motive force of speciation. Bioessays, 31(6), 642-650. https://doi.org/10.1002/bies.200800139

Grunau, C., Voigt, S., Dobler, R., Dowling, D., \& Reinhardt, K. (2018). The Cytoplasm Affects the Epigenome in Drosophila melanogaster. Epigenomes, 2(3), 17. https://doi.org/10.3390/epigenomes2030017

Gudiseva, H. V., Pistilli, M., Salowe, R., Singh, L. N., Collins, D. W., Cole, B., ... \& Addis, V. (2019). The association of mitochondrial DNA haplogroups with POAG in African Americans. Experimental Eye Research, 181, 85-89. https://doi.org/10.1016/j.exer.2019.01.015

Haddad, R., Meter, B., \& Ross, J. A. (2018). The genetic architecture of intra-species hybrid mito-nuclear epistasis. Frontiers in Genetics, 9. https://10.3389/fgene.2018.00481 
Havird, J. C., Forsythe, E. S., Williams, A. M., Werren, J. H., Dowling, D. K., \& Sloan, D. B. (2019). Selfish mitonuclear conflict. Current Biology, 29(11), R496-R511. https://doi.org/10.1016/j.cub.2019.03.020

Havird, J. C., Hall, M. D., \& Dowling, D. K. (2015). The evolution of sex: a new hypothesis based on mitochondrial mutational erosion: mitochondrial mutational erosion. Bioessays, 37(9), 951-958. https://doi.org/10.1002/bies.201500057

Healy, T. M., \& Burton, R. S. (2019). Mitochondrial DNA has strong selective effects across the nuclear genome. bioRxiv, 643056. https://doi.org/10.1101/643056

Hill, G. E. (2015). Mitonuclear ecology. Molecular Biology and Evolution, 32(8), 19171927.https://doi.org/10.1093/molbev/msv104

Hill, G. E. (2016). Mitonuclear coevolution as the genesis of speciation and the mitochondrial DNA barcode gap. Ecology and Evolution, 6(16), 5831-5842. https://doi.org/10.1002/ece3.2338

Hill, G. E. (2017). The mitonuclear compatibility species concept. The Auk: Ornithological Advances, 134(2), 393-409. https://doi.org/10.1642/AUK-16-201.1

Hill, G. E., Havird, J. C., Sloan, D. B., Burton, R. S., Greening, C., \& Dowling, D. K. (2019). Assessing the fitness consequences of mitonuclear interactions in natural populations. Biological Reviews, 94(3), 1089-1104. https://doi.org/10.1111/brv.12493

Hill, G. E., \& Johnson, J. D. (2013). The mitonuclear compatibility hypothesis of sexual selection. Proceedings of the Royal Society B: Biological Sciences, 280(1768), 20131314. https://doi.org/10.1098/rspb.2013.1314

Hoekstra, L. A., Siddiq, M. A., \& Montooth, K. L. (2013). Pleiotropic effects of a mitochondrial-nuclear incompatibility depend upon the accelerating effect of temperature in Drosophila. Genetics, 195(3), 1129-1139. https://doi.org/10.1534/genetics.113.154914

Hoekstra, L. A., Julick, C. R., Mika, K. M., \& Montooth, K. L. (2018). Energy demand and the context-dependent effects of genetic interactions underlying metabolism. Evolution Letters, 2(2), 102-113. https://doi.org/10.1002/ev13.47

Holmbeck, M. A., Donner, J. R., Villa-Cuesta, E., \& Rand, D. M. (2015). A Drosophila model for mito-nuclear diseases generated by an incompatible interaction between tRNA and tRNA synthetase. Disease Models \& Mechanisms, 8(8), 843-854. https://doi.org/10.1242/dmm.019323

Horan, M. P., Gemmell, N. J., \& Wolff, J. N. (2013). From evolutionary bystander to master manipulator: the emerging roles for the mitochondrial genome as a modulator of nuclear gene expression. European Journal of Human Genetics, 21(12), 1335. https://doi.org/10.1038/ejhg.2013.75

Hudson, R. R., Slatkin, M., \& Maddison, W. P. (1992). Estimation of levels of gene flow from DNA sequence data. Genetics, 132(2), 583-589.

Immonen, E., Collet, M., Goenaga, J., \& Arnqvist, G. (2016). Direct and indirect genetic effects of sex-specific mitonuclear epistasis on reproductive ageing. Heredity, 116(3), 338. https://doi.org/10.1038/hdy.2015.112

Innocenti, P., Morrow, E. H., \& Dowling, D. K. (2011). Experimental evidence supports a sex-specific selective sieve in mitochondrial genome evolution. Science, 332(6031), 845-848. https://doi.org/10.1126/science.1201157

James, A. C., \& Ballard, J. W. O. (2003). Mitochondrial genotype affects fitness in Drosophila simulans. Genetics, 164(1), 187-194.

James, J. E., Piganeau, G., \& Eyre-Walker, A. (2016). The rate of adaptive evolution in animal mitochondria. Molecular Ecology, 25(1), 67-78. https://doi.org/10.1111/mec.13475 
Jelić, M., Arnqvist, G., Novičić, Z. K., Kenig, B., Tanasković, M., Anđelković, M., \& Stamenković-Radak, M. (2015). Sex-specific effects of sympatric mitonuclear variation on fitness in Drosophila subobscura. BMC Evolutionary Biology, 15(1), 135. https://doi.org/10.1186/s12862-015-0421-2

Jhuang, H. Y., Lee, H. Y., \& Leu, J. Y. (2017). Mitochondrial-nuclear co-evolution leads to hybrid incompatibility through pentatricopeptide repeat proteins. EMBO Reports, 18(1), 87-101. https://doi.org/10.15252/embr.201643311

Ji, F., Sharpley, M. S., Derbeneva, O., Alves, L. S., Qian, P., Wang, Y., ... \& Simon, M. (2012). Mitochondrial DNA variant associated with Leber hereditary optic neuropathy and high-altitude Tibetans. Proceedings of the National Academy of Sciences, 109(19), 7391-7396. https://doi.org/10.1073/pnas.1202484109

Kenyon, L., \& Moraes, C. T. (1997). Expanding the functional human mitochondrial DNA database by the establishment of primate xenomitochondrial cybrids. Proceedings of the National Academy of Sciences, 94(17), 9131-9135. https://doi.org/10.1073/pnas.94.17.9131

Kimber, C. M., \& Chippindale, A. K. (2013). Mutation, condition, and the maintenance of extended lifespan in Drosophila. Current Biology, 23(22), 2283-2287. https://doi.org/10.1016/j.cub.2013.09.049

Kofler, R., Pandey, R. V., \& Schlötterer, C. (2011). PoPoolation2: identifying differentiation between populations using sequencing of pooled DNA samples (Pool-Seq). Bioinformatics, 27(24), 3435-3436. https://doi.org/10.1093/bioinformatics/btr589

Kopinski, P. K., Janssen, K. A., Schaefer, P. M., Trefely, S., Perry, C. E., Potluri, P., ... \& Doan, M. T. (2019). Regulation of nuclear epigenome by mitochondrial DNA heteroplasmy. Proceedings of the National Academy of Sciences, 116(32), 1602816035. https://doi.org/10.1073/pnas.1906896116

Lajbner, Z., Pnini, R., Camus, M. F., Miller, J., \& Dowling, D. K. (2018). Experimental evidence that thermal selection shapes mitochondrial genome evolution. Scientific Reports, 8(1), 9500. https://doi.org/10.1038/s41598-018-27805-3

Lane, N. (2011). Mitonuclear match: optimizing fitness and fertility over generations drives ageing within generations. Bioessays, 33(11), 860-869. https://doi.org/10.1002/bies.201100051

Latorre-Pellicer, A., Moreno-Loshuertos, R., Lechuga-Vieco, A. V., Sánchez-Cabo, F., Torroja, C., Acín-Pérez, R., ... \& Bernad-Miana, M. L. (2016). Mitochondrial and nuclear DNA matching shapes metabolism and healthy ageing. Nature, 535(7613), 561. https://doi.org/10.1038/nature18618

Lee, H. Y., Chou, J. Y., Cheong, L., Chang, N. H., Yang, S. Y., \& Leu, J. Y. (2008). Incompatibility of nuclear and mitochondrial genomes causes hybrid sterility between two yeast species. Cell, 135(6), 1065-1073. https://doi.org/10.1016/j.cell.2008.10.047

Levin, L., Blumberg, A., Barshad, G., \& Mishmar, D. (2014). Mito-nuclear co-evolution: the positive and negative sides of functional ancient mutations. Frontiers in Genetics, 5, 448. https://doi.org/10.3389/fgene.2014.00448

Li, H. (2013). Aligning sequence reads, clone sequences and assembly contigs with BWAMEM. arXiv preprint arXiv:1303.3997.

Lima, T. G., Burton, R. S., \& Willett, C. S. (2019). Genomic scans reveal multiple mitonuclear incompatibilities in population crosses of the copepod Tigriopus californicus. Evolution, 73(3), 609-620. https://doi.org/10.1111/evo.13690

Lunter, G., \& Goodson, M. (2011). Stampy: a statistical algorithm for sensitive and fast mapping of Illumina sequence reads. Genome Research, 21(6), 936-939. https://doi.org/10.1101/gr.111120.110 
Ma, H., Gutierrez, N. M., Morey, R., Van Dyken, C., Kang, E., Hayama, T., ... \& Laurent, L. C. (2016). Incompatibility between nuclear and mitochondrial genomes contributes to an interspecies reproductive barrier. Cell Metabolism, 24(2), 283-294. https://doi.org/10.1016/j.cmet.2016.06.012

MacLellan, K., Whitlock, M. C., \& Rundle, H. D. (2009). Sexual selection against deleterious mutations via variable male search success. Biology Letters, 5(6), 795-797. https://doi.org/10.1098/rsbl.2009.0475

Maistrenko, O. M., Serga, S. V., Vaiserman, A. M., \& Kozeretska, I. A. (2016). Longevitymodulating effects of symbiosis: insights from Drosophila-Wolbachia interaction. Biogerontology, 17(5-6), 785-803. https://doi.org/10.1007/s10522-016-9653-9

Manolio, T. A., Collins, F. S., Cox, N. J., Goldstein, D. B., Hindorff, L. A., Hunter, D. J., ... \& Cho, J. H. (2009). Finding the missing heritability of complex diseases. Nature, 461(7265), 747. https://doi.org/10.1038/nature08494

Martikainen, M. H., Grady, J. P., Ng, Y. S., Alston, C. L., Gorman, G. S., Taylor, R. W., ... \& Turnbull, D. M. (2017). Decreased male reproductive success in association with mitochondrial dysfunction. European Journal of Human Genetics, 25(10), 1162. https://doi.org/10.1038/ejhg.2017.114

McKenzie, M., Chiotis, M., Pinkert, C. A., \& Trounce, I. A. (2003). Functional respiratory chain analyses in murid xenomitochondrial cybrids expose coevolutionary constraints of cytochrome b and nuclear subunits of complex III. Molecular Biology and Evolution, 20(7), 1117-1124.

McKenzie, J.L., Chung, D.J., Healy, T.M., Brennan, R.S., Bryant, H.J., Whitehead, A. and Schulte, P.M., 2019. Mitochondrial ecophysiology: assessing the evolutionary forces that shape mitochondrial variation. Integrative and Comparative Biology, 59(4), pp.925-937. https://doi.org/10.1093/molbev/msg132

McKenzie, M., Lazarou, M., Thorburn, D. R., \& Ryan, M. T. (2007). Analysis of mitochondrial subunit assembly into respiratory chain complexes using Blue Native polyacrylamide gel electrophoresis. Analytical Biochemistry, 364(2), 128-137. https://doi.org/10.1016/j.ab.2007.02.022

Meiklejohn, C. D., Holmbeck, M. A., Siddiq, M. A., Abt, D. N., Rand, D. M., \& Montooth, K. L. (2013). An incompatibility between a mitochondrial tRNA and its nuclearencoded tRNA synthetase compromises development and fitness in Drosophila. PLoS genetics, 9(1), e1003238. https://doi.org/10.1371/journal.pgen.1003238

Meiklejohn, C. D., Montooth, K. L., \& Rand, D. M. (2007). Positive and negative selection on the mitochondrial genome. Trends in Genetics, 23(6), 259-263. https://doi.org/10.1016/j.tig.2007.03.008

Milot, E., Moreau, C., Gagnon, A., Cohen, A. A., Brais, B., \& Labuda, D. (2017). Mother's curse neutralizes natural selection against a human genetic disease over three centuries. Nature Ecology \& Evolution, 1(9), 1400. https://doi.org/10.1038/s41559017-0276-6

Mishmar, D., Ruiz-Pesini, E., Golik, P., Macaulay, V., Clark, A. G., Hosseini, S., ... \& Sukernik, R. I. (2003). Natural selection shaped regional mtDNA variation in humans. Proceedings of the National Academy of Sciences, 100(1), 171-176. https://doi.org/10.1073/pnas.0136972100

Montooth, K. L., Dhawanjewar, A. S., \& Meiklejohn, C. D. (2019). Temperature-sensitive reproduction and the physiological and evolutionary potential for Mother's Curse. Integrative and Comparative Biology. https://doi.org/10.1093/icb/icz091

Montooth, K. L., Meiklejohn, C. D., Abt, D. N., \& Rand, D. M. (2010). Mitochondrialnuclear epistasis affects fitness within species but does not contribute to fixed 
incompatibilities between species of Drosophila. Evolution, 64(12), 3364-3379. https://doi.org/10.1111/j.1558-5646.2010.01077.x

Morales, H. E., Pavlova, A., Amos, N., Major, R., Kilian, A., Greening, C., \& Sunnucks, P. (2018). Concordant divergence of mitogenomes and a mitonuclear gene cluster in bird lineages inhabiting different climates. Nature Ecology \& Evolution, 2(8), 1258. https://doi.org/10.1038/s41559-018-0606-3

Morrow, E. H., Reinhardt, K., Wolff, J. N., \& Dowling, D. K. (2015). Risks inherent to mitochondrial replacement. EMBO Reports, 16(5), 541-544. https://doi.org/10.15252/embr.201439110

Mossman, J. A., Biancani, L. M., Zhu, C. T., \& Rand, D. M. (2016a). Mitonuclear epistasis for development time and its modification by diet in Drosophila. Genetics, 203(1), 463-484. https://doi.org/10.1534/genetics.116.187286

Mossman, J. A., Jennifer, Y. G., Navarro, F., \& Rand, D. M. (2019). Mitochondrial DNA fitness depends on nuclear genetic background in Drosophila. G3: Genes, Genomes, Genetics, 9(4), 1175-1188. https://doi.org/10.1534/g3.119.400067

Mossman, J. A., Tross, J. G., Jourjine, N. A., Li, N., Wu, Z., \& Rand, D. M. (2017). Mitonuclear interactions mediate transcriptional responses to hypoxia in Drosophila. Molecular Biology and Evolution, 34(2), 447-466. https://doi.org/10.1093/molbev/msw246

Mossman, J. A., Tross, J. G., Li, N., Wu, Z., \& Rand, D. M. (2016b). Mitochondrial-nuclear interactions mediate sex-specific transcriptional profiles in Drosophila. Genetics, 204(2), 613-630. https://doi.org/10.1534/genetics.116.192328

Moya, A., Peretó, J., Gil, R., \& Latorre, A. (2008). Learning how to live together: genomic insights into prokaryote-animal symbioses. Nature Reviews Genetics, 9(3), 218. https://doi.org/10.1038/nrg2319

Nagao, Y., Totsuka, Y., Atomi, Y., Kaneda, H., Lindahl, K. F., Imai, H., \& Yonekawa, H. (1998). Decreased physical performance of congenic mice with mismatch between the nuclear and the mitochondrial genome. Genes \& Genetic systems, 73(1), 21-27. https://doi.org/10.1266/ggs.73.21

Radha, V. N., Aitkenhead, I., Clancy, D., Chown, S., \& Dowling, D. K. (2019 in-press). Sexspecific effects of mitochondrial haplotype on metabolic rate in Drosophila melanogaster support predictions of the Mother's Curse hypothesis. Philosophical Transactions of the Royal Society B: Biological Sciences.

Nakada, K., Sato, A., Yoshida, K., Morita, T., Tanaka, H., Inoue, S. I., ... \& Hayashi, J. I. (2006). Mitochondria-related male infertility. Proceedings of the National Academy of Sciences, 103(41), 15148-15153. https://doi.org/10.1073/pnas.0604641103

Osada, N., \& Akashi, H. (2011). Mitochondrial-nuclear interactions and accelerated compensatory evolution: evidence from the primate cytochrome c oxidase complex. Molecular Biology and Evolution, 29(1), 337-346. https://doi.org/10.1093/molbev/msr211

Partridge, L., \& Andrews, R. (1985). The effect of reproductive activity on the longevity of male Drosophila melanogaster is not caused by an acceleration of ageing. Journal of Insect Physiology, 31(5), 393-395. https://doi.org/10.1016/0022-1910(85)90084-8

Patel, M. R., Miriyala, G. K., Littleton, A. J., Yang, H., Trinh, K., Young, J. M., ... \& Malik, H. S. (2016). A mitochondrial DNA hypomorph of cytochrome oxidase specifically impairs male fertility in Drosophila melanogaster. Elife, 5, e16923. https://doi.org/10.7554/eLife.16923.001

Pichaud, N., Bérubé, R., Côté, G., Belzile, C., Dufresne, F., Morrow, G., ... \& Blier, P. (2019). How much mitonuclear mismatch can mitochondria tolerate?. Frontiers in Genetics, 10, 130. https://doi.org/10.3389/fgene.2019.00130 
Popadin, K. Y., Nikolaev, S. I., Junier, T., Baranova, M., \& Antonarakis, S. E. (2012). Purifying selection in mammalian mitochondrial protein-coding genes is highly effective and congruent with evolution of nuclear genes. Molecular Bology and Evolution, 30(2), 347-355. https://doi.org/10.1093/molbev/mss219

Priest, N. K., Roach, D. A., \& Galloway, L. F. (2007). Mating-induced recombination in fruit flies. Evolution, 61(1), 160-167. https://doi.org/10.1111/j.1558-5646.2007.00013.x

Pesole, G., Allen, J. F., Lane, N., Martin, W., Rand, D. M., Schatz, G., \& Saccone, C. (2012). The neglected genome. EMBO Reports, 13(6), 473-474. https://doi.org/10.1038/embor.2012.57

R Development Core Team. (2013). R: A language and environment for statistical computing.

Radzvilavicius, A. L. (2016). Evolutionary dynamics of cytoplasmic segregation and fusion: mitochondrial mixing facilitated the evolution of sex at the origin of eukaryotes. Journal of Theoretical Biology, 404, 160-168. https://doi.org/10.1016/j.jtbi.2016.05.037

Radzvilavicius, A. L., \& Blackstone, N. W. (2015). Conflict and cooperation in eukaryogenesis: implications for the timing of endosymbiosis and the evolution of sex. Journal of the Royal Society Interface, 12(111), 20150584. https://doi.org/10.1098/rsif.2015.0584

Ramsey, A. J., McCauley, D. E., \& Mandel, J. R. (2019). Heteroplasmy and patterns of cytonuclear linkage disequilibrium in wild carrot. Integrative and Comparative Biology. https://doi.org/10.1093/icb/icz102

Rand, D. M. (2001). The units of selection on mitochondrial DNA. Annual Review of Ecology and Systematics, 32(1), 415-448. https://doi.org/10.1146/annurev.ecolsys.32.081501.114109

Rand, D. M., Fry, A., \& Sheldahl, L. (2006). Nuclear-mitochondrial epistasis and Drosophila aging: Introgression of Drosophila simulans mtDNA modifies longevity in $D$. melanogaster nuclear backgrounds. Genetics, 172(1), 329-341. https://doi.org/10.1534/genetics. 105.046698

Rand, D. M., Haney, R. A., \& Fry, A. J. (2004). Cytonuclear coevolution: the genomics of cooperation. Trends in Ecology \& Evolution, 19(12), 645-653. https://doi.org/10.1016/j.tree.2004.10.003

Rand, D. M., Mossman, J. A., Zhu, L., Biancani, L. M., \& Ge, J. Y. (2018). Mitonuclear epistasis, genotype-by-environment interactions, and personalized genomics of complex traits in Drosophila. IUBMB Life, 70(12), 1275-1288. https://doi.org/10.1002/iub.1954

Rice, W. R., Linder, J. E., Friberg, U., Lew, T. A., Morrow, E. H., \& Stewart, A. D. (2005). Inter-locus antagonistic coevolution as an engine of speciation: assessment with hemiclonal analysis. Proceedings of the National Academy of Sciences, 102(suppl 1), 6527-6534. https://doi.org/10.1073/pnas.0501889102

Richardson, M. F., Weinert, L. A., Welch, J. J., Linheiro, R. S., Magwire, M. M., Jiggins, F. M., \& Bergman, C. M. (2012). Population genomics of the Wolbachia endosymbiont in Drosophila melanogaster. PLoS Genetics, 8(12), e1003129. https://doi.org/10.1371/journal.pgen.1003129

Roubertoux, P. L., Sluyter, F., Carlier, M., Marcet, B., Maarouf-Veray, F., Chérif, C., ... \& Verrier, B. (2003). Mitochondrial DNA modifies cognition in interaction with the nuclear genome and age in mice. Nature Genetics, 35(1), 65.

https://doi.org/10.1038/ng1230 
Ruiz-Pesini, E., Mishmar, D., Brandon, M., Procaccio, V., \& Wallace, D. C. (2004). Effects of purifying and adaptive selection on regional variation in human mtDNA. Science, 303(5655), 223-226. https://doi.org/10.1126/science.1088434

Sackton, Timothy B., Robert A. Haney, and David M. Rand. (2003). "Cytonuclear coadaptation in Drosophila: disruption of cytochrome c oxidase activity in backcross genotypes." Evolution 57(10), 2315-2325. https://doi.org/10.1111/j.00143820.2003.tb00243.x

Salvucci, E. (2016). Microbiome, holobiont and the net of life. Critical Reviews in Microbiology, 42(3), 485-494. https://doi.org/10.3109/1040841X.2014.962478

Seong, S. Y., Choi, M. S., \& Kim, I. S. (2001). Orientia tsutsugamushi infection: overview and immune responses. Microbes and Infection, 3(1), 11-21. https://doi.org/10.1016/S1286-4579(00)01352-6

Shtolz, N., \& Mishmar, D. (2019). The mitochondrial genome-on selective constraints and signatures at the organism, cell, and single mitochondrion levels. Frontiers in Ecology and Evolution, 7, 342. https://doi.org/10.3389/fevo.2019.00342

Sloan, D. B., Havird, J. C., \& Sharbrough, J. (2017). The on-again, off-again relationship between mitochondrial genomes and species boundaries. Molecular Ecology, 26(8), 2212-2236. https://doi.org/10.1111/mec.13959

Sloan, D. B., Triant, D. A., Forrester, N. J., Bergner, L. M., Wu, M., \& Taylor, D. R. (2014). A recurring syndrome of accelerated plastid genome evolution in the angiosperm tribe Sileneae (Caryophyllaceae). Molecular Phylogenetics and Evolution, 72, 82-89. https://doi.org/10.1016/j.ympev.2013.12.004

Smith, S., Turbill, C., \& Suchentrunk, F. (2010). Introducing mother's curse: low male fertility associated with an imported mtDNA haplotype in a captive colony of brown hares. Molecular Ecology, 19(1), 36-43. https://doi.org/10.1111/j.1365294X.2009.04444.X

Solignac, M. (2004). Mitochondrial DNA in the Drosophila melanogaster complex. Genetica, 120, 41-50.

St John, J. C. (2019). Genomic balance: two genomes establishing synchrony to modulate cellular fate and function. Cells, 8(11), 1306. https://doi.org/10.3390/cells8111306

Stojković, B., \& Đorđević, M. (2017). Interaction between mitochondrial and nuclear genomes: the role in life-history evolution. Biologia Serbica, 39(1), 32-40. https://doi.org/10.5281/zenodo.826619

Taanman, J. W. (1999). The mitochondrial genome: structure, transcription, translation and replication. Biochimica et Biophysica Acta (BBA)-Bioenergetics, 1410(2), 103-123. https://doi.org/10.1016/S0005-2728(98)00161-3

Tobler, M., Barts, N., \& Greenway, R. (2019). Mitochondria and the origin of species: bridging genetic and ecological perspectives on speciation processes. Integrative and comparative Biology. https://doi.org/10.1093/icb/icz025

Tourmente, M., Hirose, M., Ibrahim, S., Dowling, D. K., Tompkins, D. M., Roldan, E. R., \& Gemmell, N. J. (2017). mtDNA polymorphism and metabolic inhibition affect sperm performance in conplastic mice. Reproduction, 154(4), 341-354. https://doi.org/10.1530/REP-17-0206

Turelli, M. (1994). Evolution of incompatibility-inducing microbes and their hosts. Evolution, 48(5), 1500-1513. https://doi.org/10.1111/j.1558-5646.1994.tb02192.x

Vaught, R. C., \& Dowling, D. K. (2018). Maternal inheritance of mitochondria: implications for male fertility?. Reproduction, 155(4), R159-R168. https://doi.org/10.1530/REP17-0600 
Wade, M. J. (2014). Paradox of mother's curse and the maternally provisioned offspring microbiome. Cold Spring Harbor Perspectives in Biology, 6(10), a017541. https://doi.org/10.1101/cshperspect.a017541

Wei, W., Tuna, S., Keogh, M. J., Smith, K. R., Aitman, T. J., Beales, P. L., ... \& Brennan, P. (2019). Germline selection shapes human mitochondrial DNA diversity. Science, 364(6442), eaau6520. https://doi.org/10.1126/science.aau6520

Werren, J. H. (1997). Biology of Wolbachia. Annual review of entomology, 42(1), 587-609. https://doi.org/10.1146/annurev.ento.42.1.587

Werren, J. H., Baldo, L., \& Clark, M. E. (2008). Wolbachia: master manipulators of invertebrate biology. Nature Reviews Microbiology, 6(10), 741. https://doi.org/10.1038/nrmicro1969

Williams, B. R., Van Heerwaarden, B., Dowling, D. K., \& Sgro, C. M. (2012). A multivariate test of evolutionary constraints for thermal tolerance in Drosophila melanogaster. Journal of Evolutionary Biology, 25(7), 1415-1426. https://doi.org/10.1111/j.14209101.2012.02536.X

Wolff, J. N., \& Gemmell, N. J. (2013). Mitochondria, maternal inheritance, and asymmetric fitness: why males die younger. BioEssays, 35(2), 93-99. https://doi.org/10.1002/bies.201200141

Wolff, J. N., Ladoukakis, E. D., Enríquez, J. A., \& Dowling, D. K. (2014). Mitonuclear interactions: evolutionary consequences over multiple biological scales. Philosophical Transactions of the Royal Society B: Biological Sciences, 369(1646), 20130443. https://doi.org/10.1098/rstb.2013.0443

Xu, H., DeLuca, S. Z., \& O'Farrell, P. H. (2008). Manipulating the metazoan mitochondrial genome with targeted restriction enzymes. Science, 321(5888), 575-577. https://doi.org/10.1126/science.1160226

Yan, Z., Ye, G. Y., \& Werren, J. (2018). Evolutionary rate coevolution between mitochondria and mitochondria-associated nuclear-encoded proteins in insects. bioRxiv, 288456. https://doi.org/10.1101/288456

Yee, W. K., Sutton, K. L., \& Dowling, D. K. (2013). In vivo male fertility is affected by naturally occurring mitochondrial haplotypes. Current Biology, 23(2), R55-R56. https://doi.org/10.1016/j.cub.2012.12.002

Zeyl, C., Andreson, B., \& Weninck, E. (2005). Nuclear-mitochondrial epistasis for fitness in Saccharomyces cerevisiae. Evolution, 59(4), 910-914. https://doi.org/10.1111/j.00143820.2005.tb01764.x

Zhu, C. T., Ingelmo, P., \& Rand, D. M. (2014). G× G× E for lifespan in Drosophila: mitochondrial, nuclear, and dietary interactions that modify longevity. PLoS Genetics, 10(5), e1004354. https://doi.org/10.1371/journal.pgen.1004354

Zhu, Z., Han, X., Wang, Y., Liu, W., Lu, Y., Xu, C., ... \& Han, C. (2019). Identification of specific nuclear genetic loci and genes that interact with the mitochondrial genome and contribute to fecundity in Caenorhabditis elegans. Frontiers in Genetics, 10, 28.

Zhu, Z., Lu, Q., Zeng, F., Wang, J., \& Huang, S. (2015). Compatibility between mitochondrial and nuclear genomes correlates with the quantitative trait of lifespan in Caenorhabditis elegans. Scientific Reports, 5, 17303.

https://doi.org/10.1038/srep17303

Zug, R., \& Hammerstein, P. (2015). Bad guys turned nice? A critical assessment of Wolbachia mutualisms in arthropod hosts. Biological Reviews, 90(1), 89-111. https://doi.org/10.1111/brv.12098 


\section{TABLES AND FIGURES}

Table 1. Mean pairwise Fst of significantly differentiated mitochondrial single nucleotide polymorphisms (SNPs) (Fisher's exact test; FDR=0.1\%). SNPs are ranked according to FST. Pairwise FST between lines (replicates were pooled) was calculated based on Hudson et al., (1992) using PoPoolation2 (Kofler et al., 2011). Means of all pairwise FST between the mitochondrial genotypes derived from each population (Australia:A, Benin:B, Canada:C). Population replicates carrying outlier mitotypes AA3, CA1 and CA3 were excluded. Superscript lettering corresponds to nucleotide changes that result in non-synonymous $\left({ }^{\mathrm{NS}}\right)$ amino acid changes and changes in non-coding RNAs $\left({ }^{\mathrm{R}}\right)$. Mitochondrial (mt) position is according to mitochondrial reference sequence NC_024511.

\begin{tabular}{|c|c|c|c|c|c|c|}
\hline \multirow[b]{2}{*}{ mt position } & \multicolumn{3}{|l|}{ Fst between } & \multicolumn{3}{|c|}{ Fst between (excl. AA3, CA1 \& CA3) } \\
\hline & $m t A \& m t B$ & mtA \& mtC & $\mathrm{mtC} \& \mathrm{mtB}$ & $m t A \& m t B$ & $m t A \& m t C$ & $\mathrm{mtC} \& \mathrm{mtB}$ \\
\hline \multicolumn{7}{|l|}{ mtA-specifc } \\
\hline \multicolumn{7}{|l|}{ SNPs: } \\
\hline 12,381 & 0.98 & 0.98 & 0.00 & 0.98 & 0.98 & 0.00 \\
\hline 1,512 & 0.98 & 0.98 & 0.00 & 0.98 & 0.99 & 0.00 \\
\hline $4616^{\text {NS }}$ & 0.98 & 0.98 & 0.00 & 0.98 & 0.98 & 0.00 \\
\hline $9065^{\text {NS }}$ & 0.97 & 0.98 & 0.00 & 0.97 & 0.98 & 0.00 \\
\hline $13561^{R}$ & 0.96 & 0.96 & 0.00 & 0.97 & 0.97 & 0.00 \\
\hline 4,247 & 0.84 & 0.84 & 0.00 & 0.99 & 0.98 & 0.00 \\
\hline 2,661 & 0.83 & 0.83 & 0.00 & 0.98 & 0.99 & 0.00 \\
\hline 12,345 & 0.83 & 0.83 & 0.00 & 0.98 & 0.98 & 0.00 \\
\hline 8,876 & 0.82 & 0.82 & 0.00 & 0.99 & 0.99 & 0.00 \\
\hline 3,583 & 0.83 & 0.83 & 0.00 & 0.98 & 0.98 & 0.00 \\
\hline 8,982 & 0.83 & 0.83 & 0.00 & 0.98 & 0.98 & 0.00 \\
\hline 5,396 & 0.82 & 0.82 & 0.00 & 0.98 & 0.98 & 0.00 \\
\hline 10,226 & 0.81 & 0.82 & 0.00 & 0.98 & 0.98 & 0.00 \\
\hline 6,989 & 0.81 & 0.81 & 0.00 & 0.97 & 0.97 & 0.00 \\
\hline 7,871 & 0.81 & 0.81 & 0.00 & 0.97 & 0.98 & 0.00 \\
\hline $6308^{R}$ & 0.80 & 0.80 & 0.00 & 0.98 & 0.98 & 0.00 \\
\hline 1,154 & 0.80 & 0.80 & 0.00 & 0.95 & 0.95 & 0.00 \\
\hline 14,917 & 0.81 & 0.80 & 0.00 & 0.94 & 0.93 & 0.00 \\
\hline 14,929 & 0.79 & 0.78 & 0.01 & 0.95 & 0.93 & 0.01 \\
\hline 15,610 & 0.70 & 0.70 & 0.00 & 0.97 & 0.96 & 0.01 \\
\hline 18,156 & 0.69 & 0.71 & 0.01 & 0.92 & 0.97 & 0.02 \\
\hline 19,482 & 0.66 & 0.67 & 0.00 & 0.81 & 0.82 & 0.01 \\
\hline 15,321 & 0.69 & 0.67 & 0.00 & 0.78 & 0.78 & 0.00 \\
\hline 15,322 & 0.64 & 0.62 & 0.01 & 0.78 & 0.76 & 0.01 \\
\hline 17,255 & 0.71 & 0.67 & 0.02 & 0.69 & 0.65 & 0.01 \\
\hline 2,262 & 0.47 & 0.47 & 0.00 & 0.62 & 0.62 & 0.00 \\
\hline 3,385 & 0.46 & 0.47 & 0.00 & 0.61 & 0.62 & 0.00 \\
\hline 7,232 & 0.46 & 0.46 & 0.00 & 0.61 & 0.62 & 0.00 \\
\hline 15,234 & 0.20 & 0.21 & 0.01 & 0.24 & 0.24 & 0.02 \\
\hline 15,243 & 0.22 & 0.22 & 0.01 & 0.18 & 0.21 & 0.01 \\
\hline \multicolumn{7}{|c|}{ mtB-specific SNPs: } \\
\hline 1,836 & 0.99 & 0.12 & 0.77 & 0.99 & 0.00 & 0.99 \\
\hline 15,149 & 0.80 & 0.08 & 0.58 & 0.77 & 0.02 & 0.76 \\
\hline $13934^{R}$ & 0.77 & 0.13 & 0.56 & 0.77 & 0.00 & 0.78 \\
\hline
\end{tabular}


bioRxiv preprint doi: https://doi.org/10.1101/842716; this version posted November 15, 2019. The copyright holder for this preprint (which was not certified by peer review) is the author/funder, who has granted bioRxiv a license to display the preprint in perpetuity. It is made available under aCC-BY-NC-ND 4.0 International license.

mtC-specific SNPs:

12,132

0.08

0.61

0.79

0.00

0.98

1.00 
Table 2. Annotation of significantly differentiated mitochondrial SNPs (Fisher's exact test; FDR=0.1\%) derived from each population (Australia:A, Benin:B, Canada:C). Population replicates carrying outlier mitotypes AA3, CA1 and CA3 were excluded. Mitochondrial (mt) position indicates the location of the SNP within the mitochondrial genome according to mitochondrial reference sequence NC_024511. Gene indicates the name of the gene the SNP is located in. Substitution indicates whether the nucleotide change results in a change in the amino acid encoded; with synonymous indicating no change, and nonsynonymous indicating a change. Most frequent SNP allele indicates the most frequent nucleotide present for a given positional location for mtDNA captured from each population. Amino acid indicates the identity of the amino acid encoded by a particular SNP.

\begin{tabular}{|c|c|c|c|c|c|c|c|c|}
\hline $\begin{array}{l}\text { mt } \\
\text { position }\end{array}$ & gene & substitution & $\begin{array}{l}\text { mtA most } \\
\text { frequent } \\
\text { SNP allele }\end{array}$ & $\begin{array}{l}\text { amino } \\
\text { acid }\end{array}$ & $\begin{array}{l}\mathrm{mtB} \text { most } \\
\text { frequent } \\
\text { SNP allele }\end{array}$ & $\begin{array}{l}\text { amino } \\
\text { acid }\end{array}$ & $\begin{array}{l}\text { mtC most } \\
\text { frequent } \\
\text { SNP allele }\end{array}$ & $\begin{array}{l}\text { amino } \\
\text { acid }\end{array}$ \\
\hline \multicolumn{9}{|l|}{$\begin{array}{l}\text { mtA- } \\
\text { specifc } \\
\text { SNPs: }\end{array}$} \\
\hline 1,154 & ND2 & synonymous & $\mathrm{T}$ & Asn & C & Asn & C & Asn \\
\hline 1,512 & $\mathrm{Col}$ & synonymous & $\mathrm{T}$ & lle & C & lle & C & lle \\
\hline 2,262 & Col & synonymous & A & Lys & G & Lys & G & Lys \\
\hline 2,661 & $\mathrm{Col}$ & synonymous & $\mathrm{T}$ & Pro & C & Pro & C & Pro \\
\hline 3,385 & Coll & synonymous & T & Gly & C & Gly & C & Gly \\
\hline 3,583 & Coll & synonymous & C & Ala & $\mathrm{T}$ & Ala & $\mathrm{T}$ & Ala \\
\hline 4,247 & ATPase6 & synonymous & $\mathrm{T}$ & Gly & C & Gly & C & Gly \\
\hline 4,616 & ATPase6 & nonsynonymous & A & Met & $\mathrm{T}$ & Ile & $\mathrm{T}$ & Ile \\
\hline 5,396 & $\begin{array}{l}\text { Colll } \\
\text { tRNA- }\end{array}$ & synonymous & $\mathrm{T}$ & Leu & C & Leu & $\mathrm{T}$ & Leu \\
\hline 6,308 & Glu-TTC & - & A & - & C & - & C & - \\
\hline 6,989 & ND5 & synonymous & A & Tyr & G & Tyr & G & Tyr \\
\hline 7,232 & ND5 & synonymous & G & Thr & A & Thr & A & Thr \\
\hline 7,871 & ND5 & synonymous & A & Phe & G & Phe & G & Phe \\
\hline 8,876 & ND4 & synonymous & G & Leu & A & Leu & A & Leu \\
\hline 8,982 & ND4 & synonymous & $T$ & Leu & C & Leu & C & Leu \\
\hline 9,065 & ND4 & nonsynonymous & C & Val & A & Leu & A & Leu \\
\hline 10,226 & ND6 & synonymous & $T$ & Leu & c & Leu & c & Leu \\
\hline 12,345 & $N D 1$ & synonymous & A & Gly & C & Gly & C & Gly \\
\hline 12,381 & $N D 1$ & synonymous & C & Met & $\mathrm{T}$ & Met & $T$ & Met \\
\hline 13,561 & $\ln R N A$ & - & C & - & $T$ & - & $\mathrm{T}$ & - \\
\hline 14,917 & ori & - & G & - & $A$ & - & A & - \\
\hline 14,929 & ori & - & A & - & G & - & G & - \\
\hline 15,234 & ori & - & A & - & $A / T^{*}$ & - & $A / T^{*}$ & - \\
\hline 15,243 & ori & - & A & - & $\mathrm{A} / \mathrm{T}^{\dagger}$ & - & $\mathrm{A} / \mathrm{T}^{\dagger}$ & - \\
\hline 15,321 & ori & - & A & - & $T$ & - & $T$ & - \\
\hline 15,322 & ori & - & A & - & $\mathrm{T}$ & - & $T$ & - \\
\hline 15,610 & ori & - & C & - & A & - & A & - \\
\hline 17,255 & ori & - & A & - & G & - & G & - \\
\hline 18,156 & ori & - & A & - & $\mathrm{T}$ & - & $T$ & - \\
\hline $\begin{array}{l}19,482 \\
\text { mtB- } \\
\text { specific } \\
\text { SNPs: }\end{array}$ & ori & - & G & - & A & - & A & - \\
\hline
\end{tabular}




\begin{tabular}{lllllllll}
1,836 & Col & synonymous & G & Gly & A & Gly & G & Gly \\
13,934 & InRNA & - & T & - & A & - & T & - \\
$\begin{array}{l}15,149 \\
\text { mtC- }\end{array}$ & ori & - & T & - & C & - & T & - \\
$\begin{array}{l}\text { specific } \\
\text { SNPs: }\end{array}$ & & & & & & & & \\
12,132 & $N D 1$ & synonymous & T & Met & T & Met & C & Met \\
\hline
\end{tabular}

${ }^{*}+$ SNP alleles at positions 15,234 and 15,243 are of intermediate frequencies. For both Benin and Canada, allele frequencies are 0.6 and 0.4 at site 15,234 for A and T, respectively; and 0.5 and 0.5 at site 15,243 . Mitochondrial $(\mathrm{mt})$ positions are according to mitochondrial reference sequence NC_024511. 
Table 3. A. Effects of genotype and sex on lifespan across the 27 cytonuclear population replicates. Linear mixed effects model of lifespan (measured in days) with fixed effects of cytoplasm (Cyto), nuclear background (Nuclear), sex (Sex) and interactions between the three. Interactions included those between cytoplasm and nuclear background (Cyto $\times$ Nuclear) cytoplasm and sex (Cyto $\times$ Sex), nuclear background and sex (Nuclear $\times$ Sex), and three-way interactions between cytoplasm, nuclear background and sex (Cyto $\times$ Nuclear $\times$ Sex $)$. Random effects were experimental block (Block), vial identity (Vial), cyto-nuclear population replicate (Replicate), the interaction between experimental block and cyto-nuclear population replicate (Block $\times$ Replicate), and the interaction between cyto-nuclear population replicate and sex (Replicate $\times$ Sex). B. Effects of experimental mismatching of cyto-nuclear genotypes on longevity. Fixed effects are coevolutionary status (Status) (coevolved or mismatched) and sex. Random effects included cytoplasm (Cyto) and nuclear background (Nuclear), any interactions between the the two and also all possible interactions with sex (Sex). Interactions of random effects included those between cytoplasm and nuclear background (Cyto $\times$ Nuclear), cytoplasm and sex $($ Cyto $\times$ Sex $)$, nuclear background and sex (Nuclear $\times$ Sex $)$ and three-way interactions between cytoplasm, nuclear background and sex $($ Cyto $\times$ Nuclear $\times$ Sex $)$. Other random effects included block (Block), vial identity (Vial), cyto-nuclear population replicate (Replicate), the interaction between experimental block and cyto-nuclear population replicate (Block $\times$ Replicate), and the interaction between cyto-nuclear population replicate and sex (Replicate $x$ Sex).

Table 3A.

\begin{tabular}{llll}
\hline Fixed effects & $X^{\mathbf{2}}$ & Df & $P$ \\
\hline Cyto & 2.7573 & 2 & 0.2519 \\
Nuclear & 3.8558 & 2 & 0.1455 \\
Sex & 2.0494 & 1 & 0.1523 \\
Cyto $\times$ Nuclear & 6.2957 & 4 & 0.1781 \\
Cyto $\times$ Sex & 5.4411 & 2 & 0.0658 \\
Nuclear $\times$ Sex & 14.020 & 2 & 0.0009 \\
Cyto $\times$ Nuclear $\times$ Sex & 11.150 & 4 & 0.0249 \\
Random effects & Variance & & \\
Block & 1.941 & & \\
Vial & 3.949 & & \\
Replicate & 2.918 & & \\
Block $\times$ Replicate & 1.397 & &
\end{tabular}


Replicate $\times$ Sex

3.497

Residual

57.376

Table 3B.

\begin{tabular}{llll}
\hline Fixed effects & $X^{2}$ & Df & $P$ \\
\hline Coevolutionary status & 0.0315 & 1 & 0.8591 \\
Sex & 17.6959 & 1 & $<0.0001$ \\
Random effects & Variance & \\
Cyto & 0.000 & \\
Nuclear & $1.039 \times 10^{-5}$ & \\
Cyto $\times$ Nuclear & 0.000 & \\
Cyto x Sex & 0.000 & \\
Nuclear x Sex & 3.196 & \\
Cyto x Nuclear x Sex & 0.642 & \\
Block & 1.939 & \\
Vial & 3.947 & \\
Replicate & 2.865 & \\
Block x Replicate & 1.400 & \\
Replicate $\times$ Sex & 3.520 & \\
\hline
\end{tabular}


Table 4. Effects of mitotype and sex on lifespan for the analysis of the Australian nuclear background only. Linear mixed effects model of longevity (measured in days) with fixed effects of mitotype identity (Mito), sex (Sex) and the interaction between mitotype and sex (Mito $\times$ Sex). Random effects were experimental block (Block), vial identity (Vial), population replicate (Replicate), the interaction between experimental block and population replicate (Block $\times$ Replicate), the interaction between population replicate and sex (Replicate $\times$ Sex), the interaction between mitotype, sex and experimental block (Mito $\times$ Nuclear $\times$ Sex), the interaction between mitotype and experimental block (Mito $\times$ Block), and the interaction between sex and experimental block (Sex $\times$ Block).

Table 4.

\begin{tabular}{|c|c|c|c|}
\hline Fixed effects & $x^{2}$ & Df & $P$ \\
\hline Mito & 2.8023 & 4 & 0.5914 \\
\hline Sex & 0.0325 & 1 & 0.8569 \\
\hline Mito x Sex & 9.4313 & 4 & 0.0512 \\
\hline Random effects & Variance & & \\
\hline Block & 1.945 & & \\
\hline Vial & 1.980 & & \\
\hline Replicate & 6.045 & & \\
\hline Block x Replicate & 1.739 & & \\
\hline Replicate x Sex & 4.047 & & \\
\hline Mito $x$ Sex x Block & 1.041 & & \\
\hline Mito x Block & 0.000 & & \\
\hline Sex x Block & 0.000 & & \\
\hline Residual & 61.946 & & \\
\hline
\end{tabular}




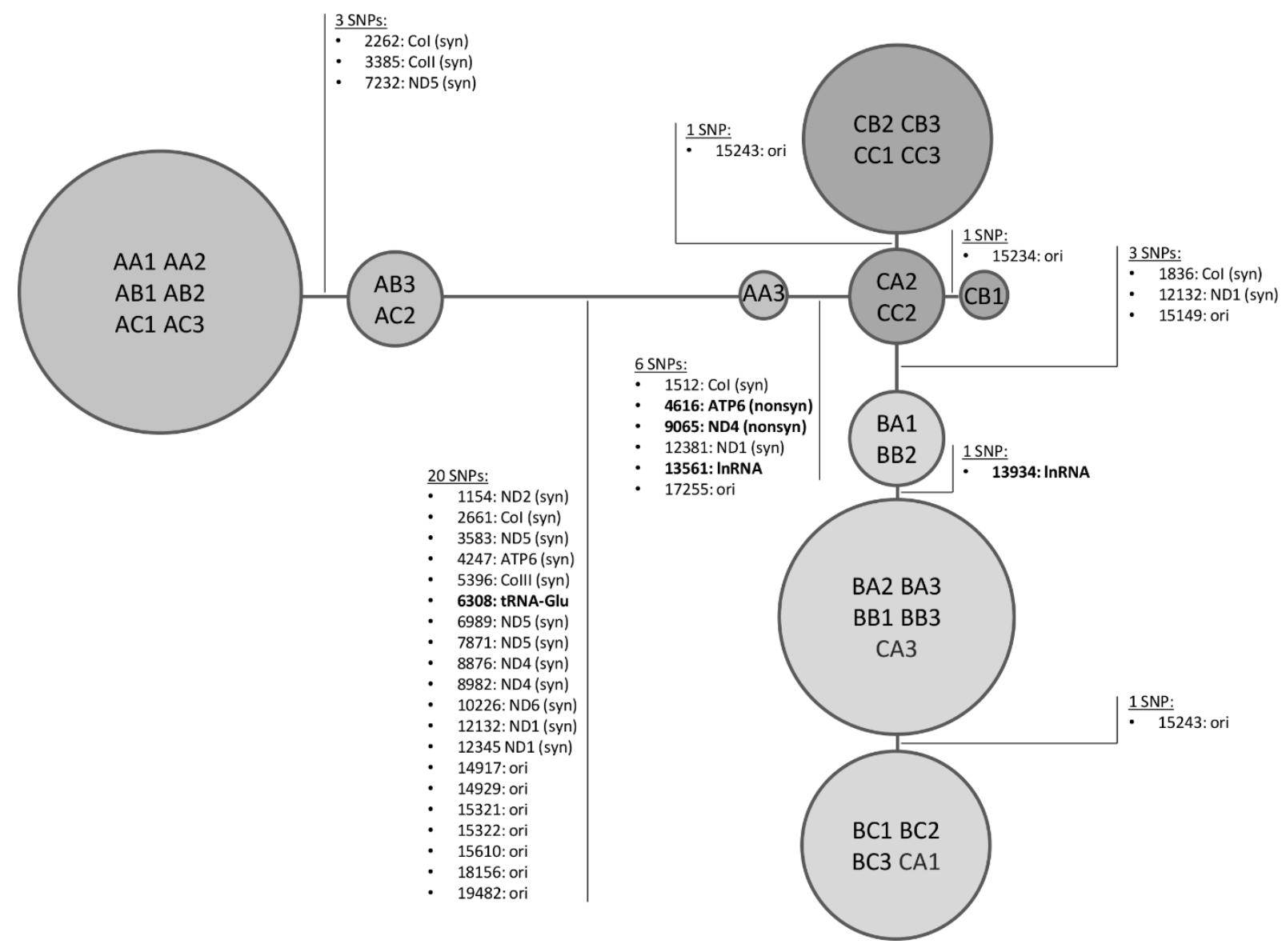

Figure 1. Network of mitochondrial genotypes of the $3 \times 3$ cyto-nuclear lines derived from the 34 significantly differentiated SNPs. The network is based on the consensus sequences of the lines. The consensus sequence of each population is defined by the most frequent nucleotide at each SNP site. Note that SNPs at positions 15,234 and 15,243 are of intermediate allele frequencies and differentiation at these two sites is relatively low (see also Table 1). 


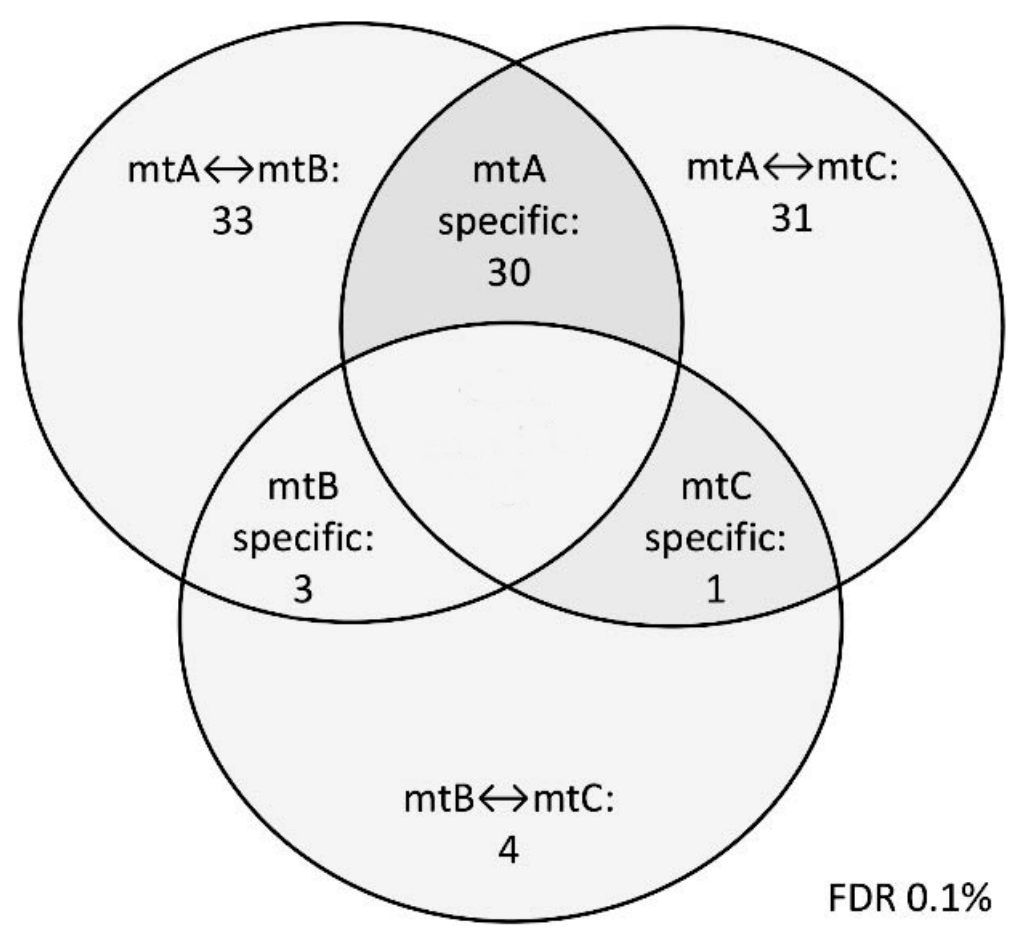

Figure 2. Diagram showing the numbers of significantly differentiated SNPs between mitochondrial genomes from Australia, Benin, and Canada. Numbers of variants specific to either Australia, Benin or Canada are given as well. Those variants are in high frequency in lines containing mtDNA derived from one origin, but are absent or in low frequency in lines with $\mathrm{mtDNA}$ from the other two origins. 


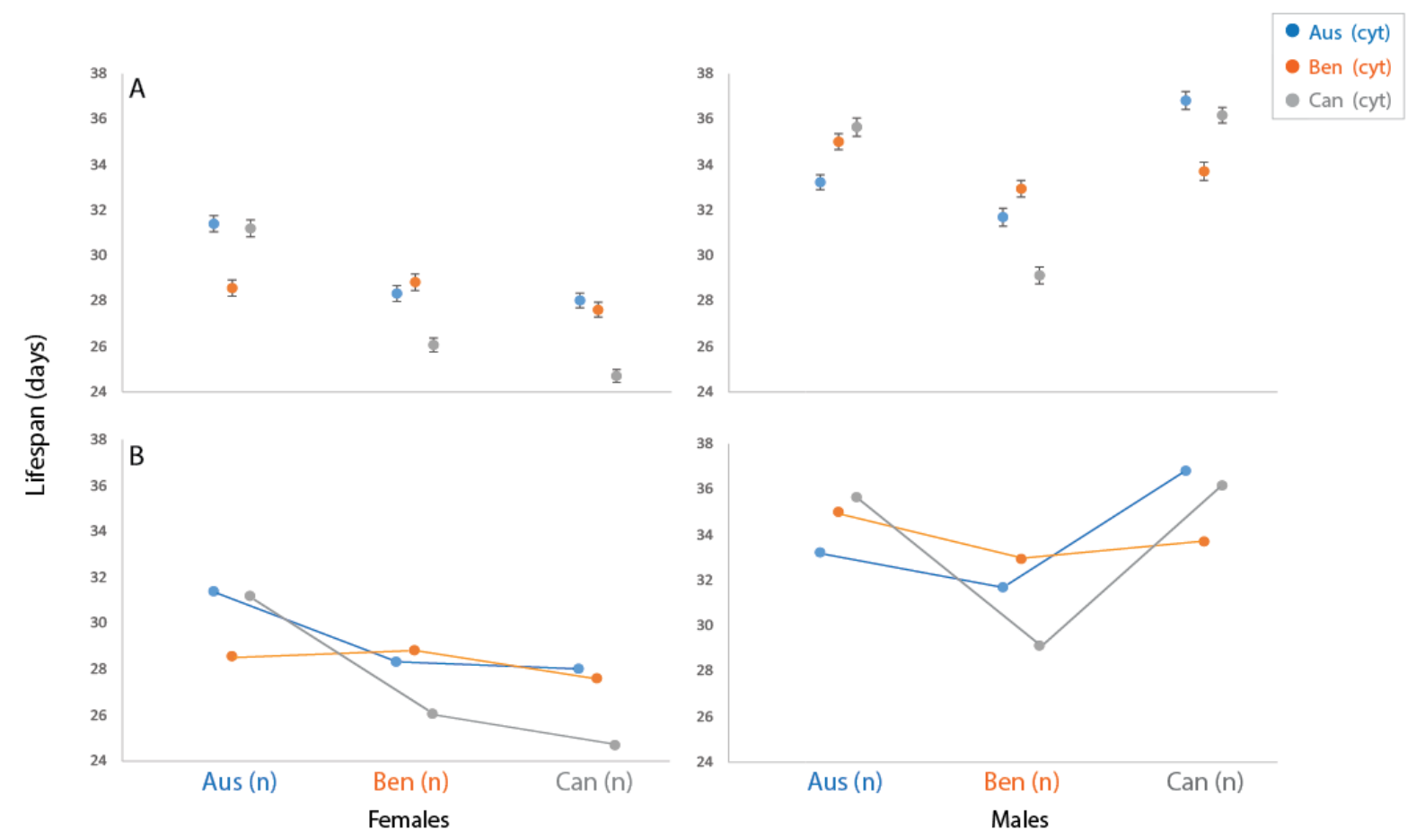

Figure 3. Cyto-nuclear effects on longevity. A. Mean lifespan ( \pm 1 standard error) of each of the three cytoplasms expressed against each of the three nuclear backgrounds, in females (left hand panel) and males (right hand panel). Lifespan (as measured in days) is indicated on the vertical axis, with the nuclear background denoted on the horizontal axis. Each colour denotes a different cytoplasm: Australia shown in blue, Benin shown in orange, Canada shown in grey. B. Reaction norms of the same data. Mean lifespan is indicated on the vertical axis, and nuclear background on the horizontal axis. 


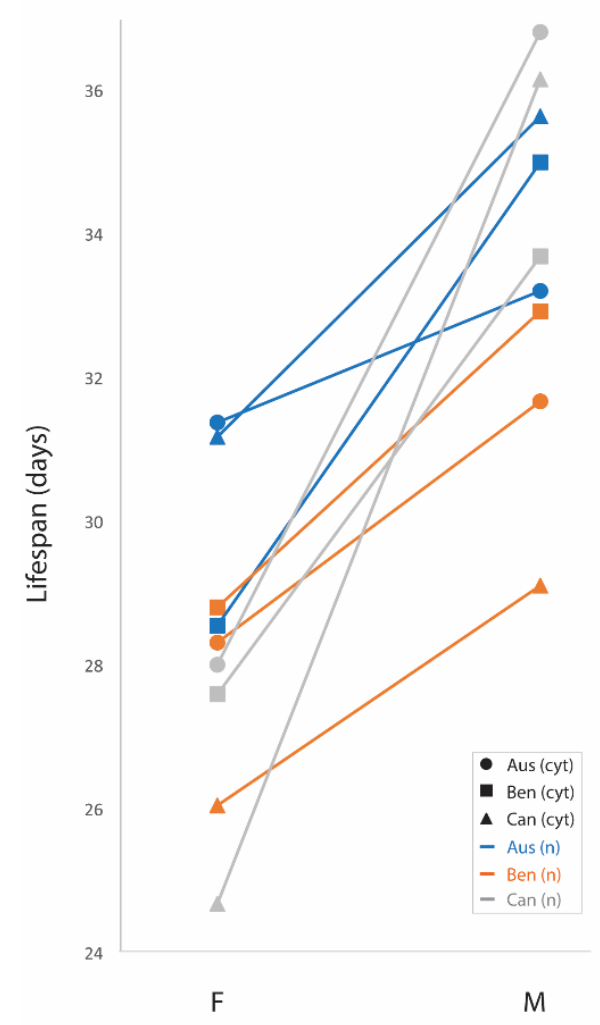

Figure 4. Performance of cyto-nuclear genotypes for the two sexes. Interaction plot of mean lifespan per sex across all cyto-nuclear genotypes. The nuclear genetic background originating from Australia is shown in blue, the nuclear genetic background originating from Benin is shown in orange, the nuclear genetic background originating from Canada is shown in grey. Cytoplasms originating from Australia shown with circles, cytoplasms originating from Benin shown with shown with squares, cytoplasms originating from Canada shown with triangles. Sexes, indicated by F or M. 


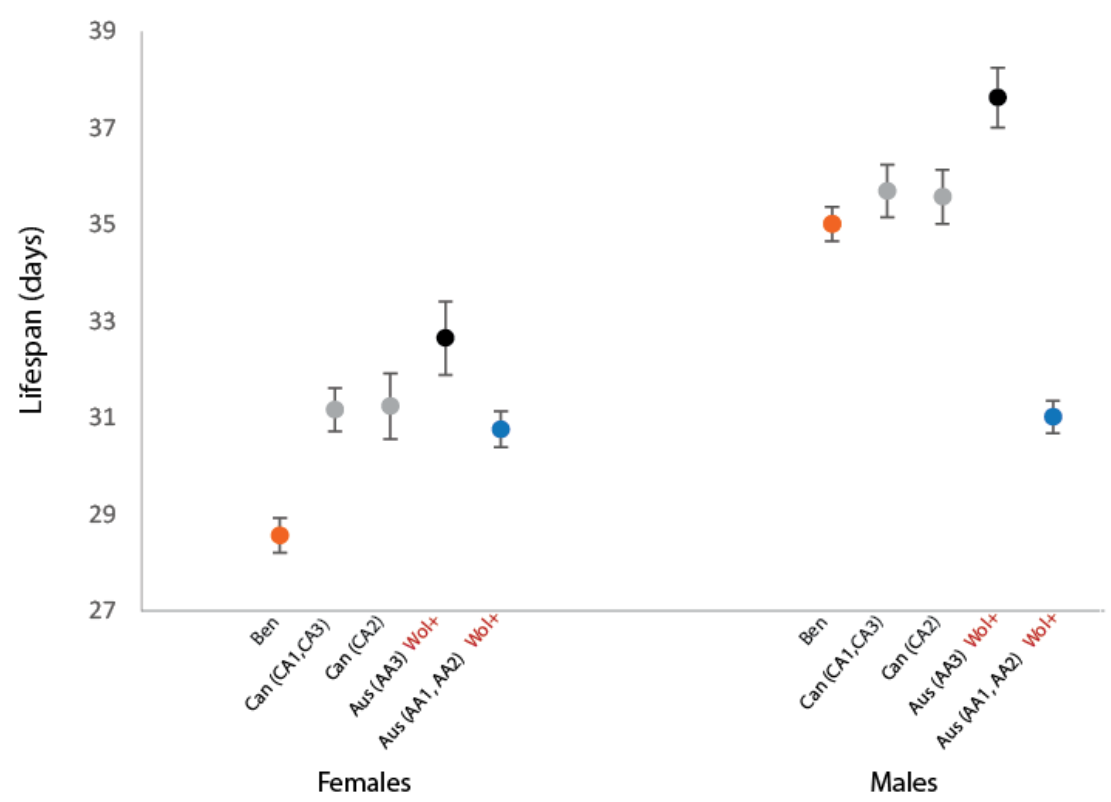

Figure 5. Mean lifespan of mitotypes when expressed alongside the Australian nuclear background, in females and males. Comparisons are: 1) Beninese (comprising BA1, BA2, BA3), 2) Canadian CA1 and CA3 (whose prevailing mitotypes were more similar to B mitotypes than to the other C mitotypes 3) Canadian CA2, 4) Australian A1 (AA3), and 5) B1 (AA1 and AA2). Beninese cytoplasms shown in orange, Canadian shown in grey, Australian lines carrying the A1 haplotype shown in black, Australian lines carrying the B1 haplotype shown in blue. Wol+ in red lettering indicates Wolbachia infected lines, all others are free from Wolbachia infection. Error bars indicate standard error based on calculated means. 